\title{
Urbane Kinokultur
}

Das Lichtspieltheater in der Großstadt 1895-1949

Wolfgang Flügel, Merve Lühr, Winfried Müller (Hg.)

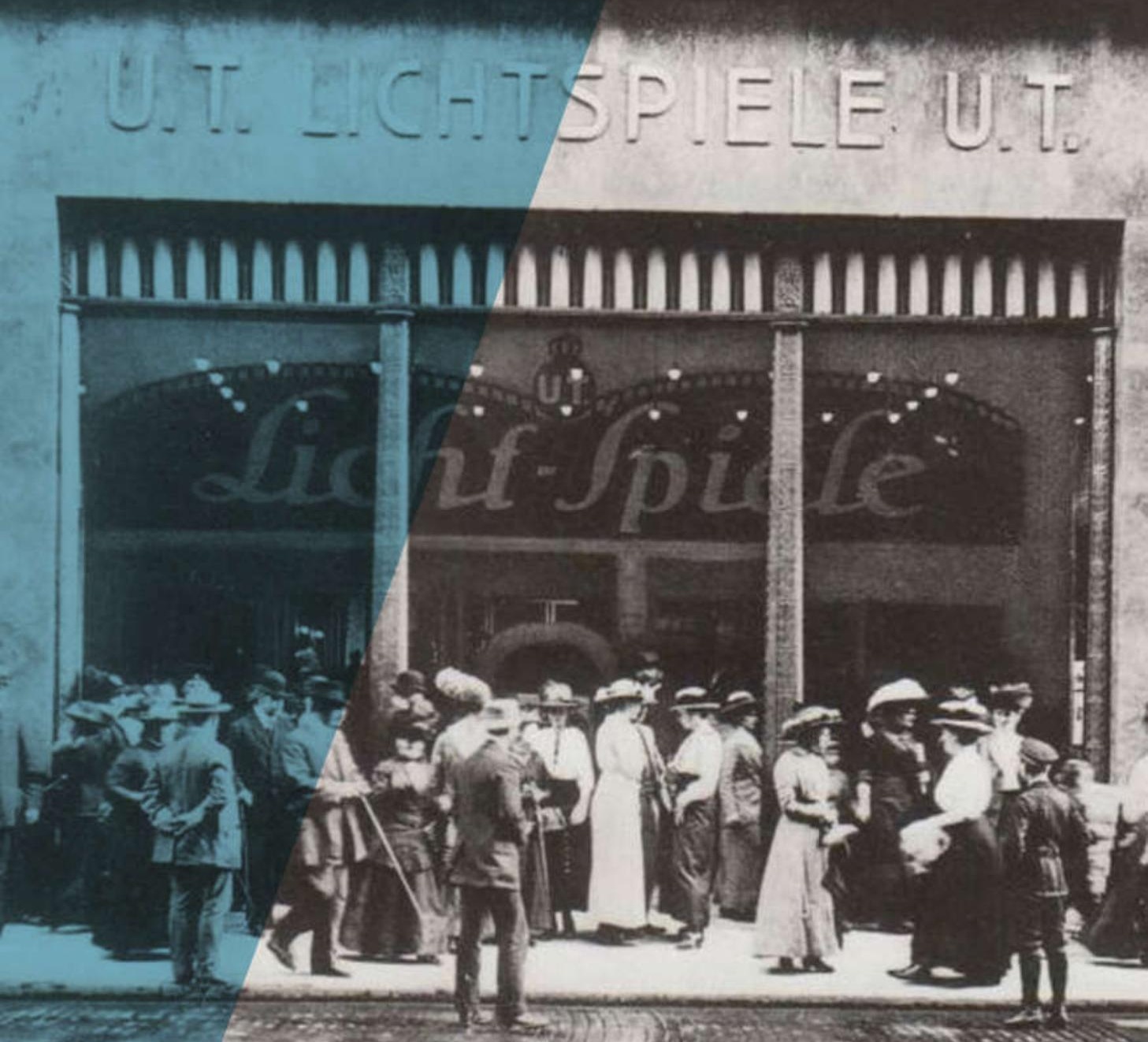





\section{Urbane Kinokultur \\ Das Lichtspieltheater in der Großstadt 1895-1949}

Wolfgang Flügel, Merve Lühr, Winfried Müller (Hg.)

in Zusammenarbeit mit Sophie Döring und Lennart Kranz 


\section{Impressum}

ISGV digital. Studien zur Landesgeschichte und Kulturanthropologie 2

herausgegeben von Enno Bünz, Andreas Rutz, Joachim Schneider und Ira Spieker

Redaktion:

Sophie Döring, Wolfgang Flügel, Merve Lühr, Winfried Müller, Susanne Müller

Layout: Josephine Rank, Berlin Technische Umsetzung (barrierefreies PDF): Klaas Posselt, einmanncombo Umschlaggestaltung: Josephine Rank nach einem Entwurf von Linda S. Gableske unter Verwendung einer Fotografie der U.T. Lichtspiele, Dresden, von 1913 (Quelle: https://filmtheater.square7.ch/ wiki/index.php?title=Datei:Dresden_UT_1913_ PK.jpg\#mw-navigation).

(C) Dresden 2020

Institut für Sächsische Geschichte und Volkskunde Zellescher Weg 17| 01069 Dresden

Bibliografische Information der Deutschen Nationalbibliothek: Die Deutsche Nationalbibliothek verzeichnet diese Publikation in der Deutschen Nationalbibliografie; detaillierte bibliografische Daten sind im Internet über http://dnb.ddb.de abrufbar.

Alle Rechte vorbehalten.

www.isgv.de

ISBN 978-3-948620-01-1

ISSN 2700-0613

DOI 10.25366/2020.41

Diese Maßnahme wird mitfinanziert durch

Steuermittel auf der Grundlage des vom

Sächsischen Landtag beschlossenen Haushaltes.

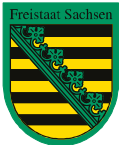




\section{Inhalt}

Wolfgang Flügel, Merve Lühr, Winfried Müller

Einleitung

Urbane Kinokultur: Das Beispiel Dresden

\section{Carola Zeh}

Bewegte Bilder - bewegte Geschichte

Zur Entwicklung des Kinos in Dresden

\section{Wolfgang Flügel}

Das frühe Dresdner Kino im Blick des Kinopioniers Heinrich Ott

\section{Sophie Döring}

Zwischen Kalklicht und Samtsessel

Mobile Kinopraxis in Sachsen 1896-1910

\section{Winfried Müller}

Ein neues Medium wird geadelt.

König Friedrich August III. von Sachsen geht ins Kino

\section{Mona Harring}

Kino- und Filmpolitik in Dresden zwischen 1945 und 1949 
Kino im urbanen Raum - Kino als urbaner Raum

\section{Lina Schröder}

Licht lockt Leute: Als der Mensch in die Schöpfung eingriff und Tag und Nacht aufhob - ein Werkstattbericht

\section{Kaspar Maase}

Kinderkino zwischen Kontrolle, Kommerz und Krawall

Anmerkungen zu einer Hamburger Initiative

aus dem frühen 20. Jahrhundert

\section{Fabian Brändle}

Wildwest und ein Schnäuzchen wie Clark Gable

Zürcher Kinokultur und Urbanität von 1900 bis 1940

\section{Sonja Neumann}

Konservenmusik und Elektrokapital

Tonfilmtechnik in München im Jahr 1929

\section{Sven Eggers}

Vor der Vorstellung

Die Herausbildung des Kinofoyers als urbane Gattung 183

\section{Merve Lühr}

Erstklassig und routiniert.

Das Lichtspieltheater als Arbeitsplatz 
Urbane Kinokultur: Die Klein- und Mittelstadt

\section{Niklas Hertwig}

„Film ab!" Max von Allweyer und seine Schulfilm-Unternehmung Lichtbildvorführungen an Volksschulen im ländlichen

Oberbayern 1926-1929

\section{Magdalena Abraham-Diefenbach}

Bellevue und Piast. Kino in den geteilten Städten

an der deutsch-polnischen Grenze 1945-1949

Jeanette Toussaint, Ralf Forster

Weltspiegel - Kino im 20. Jahrhundert.

Ein Ausstellungsprojekt

\section{Andrea Graf}

Publikum, Popcorn und Programm in der Provinz. Wie Kinokultur im ländlichen Raum funktioniert - 
| Urbane Kinokultur:

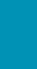

Das Beispiel Dresden

Das Beispiel Dresden

Das Beispiel Dresden

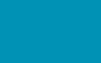

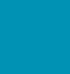

O

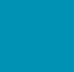

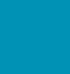

O

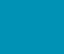




\title{
Das frühe Dresdner Kino im Blick des Kinopioniers Heinrich Ott
}

\author{
Wolfgang Flügel
}

Der Film ,Die schwarze Milch', in welchem eine schwarze Amme ein Kind stillte, wurde mir in meinem Theater, Freiberger Platz 37, verboten, da diese Szene nach Ansicht der Beamten als sittlich verrohend galt. [...] Dieses und vieles andere gab mir die Veranlassung, die Kinobesitzer von Dresden und Umgebung zu einer Aussprache nach Dresden ins Stadtkaffee einzuberufen, bei der beschlossen wurde, eine Vereinigung der Dresdner Kinematographenbesitzer zu gründen. ${ }^{1}$ Dieses Zitat führt zurück in das Jahr 1907 und damit in die Anfangszeit des Kinos. Sein Verfasser, der Kinopionier Heinrich Ott, zählte zu den wichtigen Figuren der frühen Dresdner

1 Stadtarchiv Dresden, 17.2.10: Sammlung Heinrich Ott zur Geschichte der Dresdner Kinematographie (im Folgenden: Sammlung Ott), Teil J: Gründung des Vereins der Dresdner Kinobesitzer, Blatt 2 und 4.
Kinogeschichte. Er hat deren Verlauf nicht nur mitgeprägt, sondern auch schriftlich dokumentiert. Das Ergebnis liegt in der sogenannten Sammlung Ott vor, die die großstädtische Kinokultur von ihren Anfängen im ausgehenden 19. Jahrhundert bis in die Mitte der 1930er-Jahre am Beispiel der sächsischen Kapitale feinmaschig dokumentiert. Genau darin liegt die Einmaligkeit dieser Quelle - nach derzeitigem Kenntnisstand existieren keine vergleichbaren Aufzeichnungen für die frühe Kinogeschichte anderer Städte. Deshalb nutzte das am Institut für Sächsische Geschichte und Volkskunde angesiedelte Projekt „1918 als Achsenjahr der Massenkultur. Kino, Filmindustrie und Filmkunstdiskurse in Dresden" anknüpfend an die Feststellung der Kulturwissenschaftler Esther Sabelus und Jens Wietschorke, wonach trotz zahlreicher Detailstudien die Geschichte des 
frühen Kinos in Deutschland nur unzureichend erschlossen ist, die Sammlung Ott als zentralen Grundstein für seine Analysen zur Frühzeit des Dresdner Kinos. ${ }^{2}$

Einen Ausgangspunkt sowohl für die Projektarbeit als auch für den vorliegenden Beitrag liefern die grundlegenden Beobachtungen etwa des Kulturwissenschaftlers Kaspar Maase und des Filmwissenschaftlers Joseph Garncarz, wonach das Kino in den westlichen Großstädten des frühen 20. Jahrhunderts zum Leitmedium einer modernen Massenkultur aufstieg und dabei selbst als Ausdruck eines Medienwandels zu verstehen ist. ${ }^{3}$ Hiervon ausgehend wird im Folgenden in einem kulturhistorischen Zugriff die Sammlung Ott hinsichtlich ihrer Aussagen zur Genese der Dresdner Kinotopografie analysiert. Dies erfolgt unter Rückgriff auf verschiedene Detailstudien, die zu zentralen Aspekten der frühen Kinogeschichte vorliegen. So rückt neben der Entstehung des Lichtspielwesens sowie dem Prozess seiner Ausbreitung im Stadtraum schon seit langem die Kinoarchitektur als eigene Bauaufgabe in den Blickpunkt der Forschung. ${ }^{4}$ Dabei ist der Kulturwissenschaftlerin Sabine Steidle zuzustimmen, die diese Herausbildung der Kinotopografie im Kontext von Urbanität als Ausdruck eines fundamentalen Wandels der räumlichen Ordnung kontextualisiert, in welchem Kinos zu Orten von Moderne werden. ${ }^{5}$ Entscheidend ist nun, dass dieser Prozess nicht nur mit technischen, wirtschaftlichen und soziokulturellen Aspekten kausal verbunden war, wie etwa die Medienwissenschaftlerin Corinna Müller anmerkt, sondern sich auch in Relation zu anderen Kulturformen vollzog. ${ }^{6}$ Aus diesen Facetten ergeben sich die auf die Herausbildung der Dresdner Kinotopografie zielenden Leitfragen, die an die Sammlung Ott gerichtet werden, wobei durch einen Abgleich mit den Dresdner Adressbüchern auch Aussagen zu den Kinobesitzern und -betreibern sowie zu deren sozialen Kontexten möglich sind.

\section{Heinrich Ott - Leben und Werk}

Völlig zu Unrecht hat Heinrich Ott noch keinen Eingang in die biografischen Nachschlagewerke gefunden, weshalb an dieser Stelle einige Anmerkungen zu Leben und Werk notwendig sind. Geboren wurde er am 1. August 1873 als Sohn eines Fuhrwerkbesitzers in Wiesbaden und starb am 30. Juni 1941 in Dresden. Aus den ersten gut 30 Jahren seines Lebens, über seine Ausbildung und seinen Einstieg in das Berufsleben gibt es leider keinerlei Informationen, ebenso ist unbekannt, wann er nach Dresden kam. Doch solche Informationslücken in der Biografie von Dresdner Kinobesitzern, die vielfach von außerhalb kamen, sind keine Seltenheit, was auf die grundsätzlich große Mobilität innerhalb des frühen Kinowesens deutet.

6 Müller: Kinematographie.

2 Siehe Sabelus/Wietschorke: Einleitung, S. 7.

3 Siehe etwa Maase: Vergnügen; Maase: Grenzen; Garncarz: Medienwandel.

4 Siehe Pabst: Lichtspieltheater; Baake: Lichtspielhausarchitektur; Zeh: Lichtspieltheater.

5 Siehe Steidle: Kinoarchitektur. 


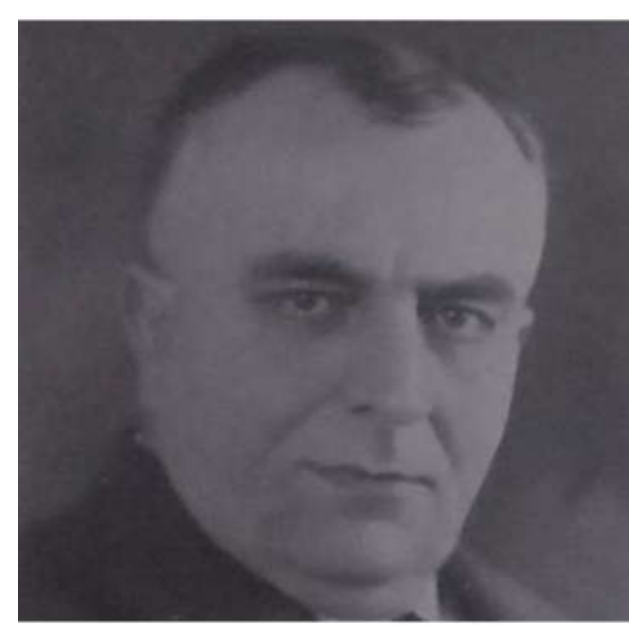

Abbildung 1: Heinrich Ott, Foto um 1929 (Quelle: Jubiläums-Festschrift, S. 29).

In der Elbmetropole wird Ott erst Ende 1906 greifbar, als er mit dem eingangs genannten $\mathrm{Ki}$ no am Freiberger Platz, dem Campaneta, eines der ersten Lichtspielhäuser in Dresden eröffnete. Bis 1932 sollte er nach und nach fünf Kinos besitzen beziehungsweise betreiben, darunter auch das Reform-Kino auf der Hygiene-Ausstellung von 1911. Mit diesem interimistischen Musterbau, den er mit allen modernen und technischen Neuheiten eines Lichtspielhauses ausstattete, demonstrierte er die Leistungsfähigkeit der noch jungen Kinobranche. Insgesamt war sein Engagement im Lichtspielbetrieb ein technisches und kaufmännisches Wagnis, das umso bemerkenswerter erscheint, als Ott und ebenso seine Ehefrau aus völlig anderen Berufsbranchen kamen: Im Hauptberuf, den er laut der

7 Sammlung Ott, Teil C: Die Kinematographie als Schaustellung, Blatt 93.
Angaben im Dresdner Adressbuch bis 1939/40 ausübte, war er Sänger (wohl Chorsänger) an der Dresdner Hofoper. Hier lernte er seine Kollegin Maria Elsa Lewandowski kennen, die er am 5. August 1909 heiratete. ${ }^{8}$

Kurz vor seiner Hochzeit hatte Ott am 14. Mai 1909 mit dem im Eingangszitat bereits erwähnten Verein der Lichtspieltheaterbesitzer von Dresden und Umgebung die wohl deutschlandweit erste Interessenvereinigung dieser Art initiiert und auch deren Leitung übernommen. ${ }^{9}$ Den Vorsitz legte er allerdings nach einem Jahr, Mitte 1910, aus beruflichen Gründen nieder. Die zahlreichen Proben an der Oper machten, so seine eigene Erklärung, eine regelmäßige Teilnahme an den Vereinssitzungen schlechterdings unmöglich. Der Verein, den die Dresdner Kinobesitzer gegründet hatten, um sich gegen willkürliche Zensur und andere als Schikanen empfundene Maßnahmen zu schützen, gehörte bald zu einem Netzwerk zahlreicher ähnlicher Interessenvereinigungen, die mit einigem Erfolg bis zum Ende der Weimarer Republik für die Belange ihrer Mitglieder eintraten..$^{10}$ Dennoch löste er sich im Mai 1934, offenkundig auf seiner Jubiläumsfeier zum 25-jährigen Bestehen, aufgrund der nationalsozialistischen Bestimmungen auf. ${ }^{11}$ Mit dieser Aussage spielt Ott auf die sogenannte Gleichschaltung an, in deren Rahmen Propagandaminister Joseph Goebbels Massenmedien und Kulturschaffende in Deutschland seiner

8 Siehe Adreßbuch für Dresden und seine Vororte, 1906-1943/44.

9 Diese Qualifizierung in Jubiläums-Festschrift, S. 7.

10 Siehe Jubiläums-Festschrift, S. 10-11 sowie S. 16-18.

11 Sammlung Ott, Teil J: Gründung des Vereins der Dresdner Kinobesitzer, Blatt 10. 
Kontrolle unterwarf und damit als Medium der nationalsozialistischen Propaganda einsetzte. Mit diesem Ziel wurde der Reichsverband Deutscher Lichtspieltheaterbesitzer als Dachverband des Kinogewerbes in die neugeschaffene und auf die politischen Ziele der Nationalsozialisten ausgerichtete Reichsfilmkammer eingegliedert. ${ }^{2}$ Die Zugehörigkeit zu dieser Institution war Pflicht für alle in der Film- und Kinobranche Tätigen. Die Zwangsmitgliedschaft bedeutete für die Kinobesitzer nicht zuletzt eine Einschränkung ihrer unternehmerischen Freiheit, da die Reichsfilmkammer sowohl in die Preis-als auch in die Programmgestaltung eingriff.

In welchem Maß Ott hiervon betroffen war, lässt sich aufgrund der dürftigen Quellenlage nicht abschätzen. Zwar hatte der Kinopionier, wohl im Kontext der Wirtschaftskrise, die auch die Filmbranche schwer traf, mit dem Theater am Bischofsplatz (T.B.-Lichtspiele) sein letztes Kino bereits im August 1932 aufgegeben. Allerdings übte er neben dem Beruf des Opernsängers bis 1938/39 auch den des Filmvertreters aus, das heißt, er vermittelte im Auftrag eines Filmverleihers Verleihverträge an Kinounternehmen, ohne selbst zum Vertragsabschluss berechtigt zu sein. ${ }^{13}$ Damit korrespondiert die ambivalente rechtliche Stellung dieser Berufsgruppe: Formal handelt es sich bei den Filmvertretern um Selbständige, tatsächlich aber war der Verleiher innen gegenüber bis hin zum Terminplan weisungsberechtigt. ${ }^{14}$ Die Frage ist, ob ein solcher Beruf die Chance bot, trotz Tätigkeit im Film- und

12 Yong: Inszenierung, S. 78.

13 Zu Otts Tätigkeit im Filmverleih siehe JubiläumsFestschrift, S. 31

14 Dienstag/Elster: Handbuch, S. 228, 230, 480.
Kinogewerbe die Zugehörigkeit zur Reichsfilmkammer zu vermeiden. Hierbei ist weitergehend auch zu fragen, ob ein Opernsänger Mitglied der Reichsmusikkammer - einer Parallelinstitution zur Reichsfilmkammer - sein musste? Die Antwort muss offenbleiben.

Festzuhalten bleibt, dass Ott zwischen 1906 und 1938 in verschiedenen Feldern der Dresdner Kinobranche tätig war. Das aus dieser dreißigjährigen Wirkungszeit gewonnene Wissen floss in die Sammlung Ott ein, die ihr Verfasser rückblickend gegen Ende seines Arbeitslebens schrieb. Die stringente Detailgenauigkeit legt nahe, dass inm eine reichhaltige Materialsammlung zur Verfügung gestanden haben muss. Doch deren Verbleib ist leider ebenso unbekannt wie die Entstehungskontexte der Sammlung Ott, für die ein Zusammenhang mit den Ereignissen des Jahres 1934 zumindest denkbar erscheint.

Das aus zwölf unterschiedlich umfangreichen Teilen bestehende Quellenkonvolut, das im Dresdner Stadtarchiv aufbewahrt wird, umfasst etwas mehr als 1.000 Seiten in Maschinenschrift. ${ }^{15}$ An zentraler Stelle steht die aus einer chronologisch geordneten und mit weiterführenden Angaben ergänzte Auflistung von 153 Dresdner Spielstätten, die zwischen 1896

15 Überliefert sind Teil A: Eine verhängnisvolle Kinovorstellung; Teil B: Dresden und die Kinematographie; Teil C: Die Kinematographie als Schaustellung; Teil D: Übersicht der Gründung der Lichtspieltheater von 1896 bis 1936 (tatsächlich endet die Übersicht im Jahr 1933); Teil E: Von dem bewegten Bild zum Tonfilm; Teil F: Dresdner Filmverleiher; Teil G: Filme einst und jetzt; Teil H: Tonfilm; Teil J: Gründung des Vereins der Dresdner Kinobesitzer; Teil K: Lustbarkeitssteuer; Teil L: Alphabetisches Verzeichnis aller Filme von 1896 bis 1936; Teil M: Chronologisches Verzeichnis aller Filme von 1896 bis 1936. 
und Ende 1933 nachweisbar sind. ${ }^{16}$ Die Namen der Besitzer, die Adresse des Kinematographen sowie das Eröffnungs- und gegebenenfalls das Schließungsdatum sind ebenso enthalten wie Informationen zum Gründungsprozess. Dabei nutzte Ott verschiedene Behördenunterlagen, die zum Teil als Kriegsverlust gelten müssen.

Einen besonderen Informationswert besitzen schließlich die den Kinolisten beigefügten Abschriften der Eröffnungsannoncen aus der Tagespresse. Sie eröffnen einen Blick in die zeitgenössische Kinowelt. So wie es zum Charakter des Kinos als Ort der populären Unterhaltung passt, dass die Eröffnungsanzeigen im Zusammenhang mit dem Kinematographen auch von Bierausschank, Tanz und von Buchverleih berichten, kündet es gleichermaßen von der Begeisterung für moderne Technik und von Sensationslust, wenn die Presse etwa von der Existenz einer 1909 noch neuartigen Rolltreppe ins Kino Imperial berichtet, von einem als sehenswerte Neuheit deklarierten elektrisch beleuchteten Eau de Cologne Springbrunnen ${ }^{17}$ - dessen Einsatz vielleicht auch die olfaktorischen Folgen mangelhafter Ventilation mindern sollte oder von einem acht Meter langen Riesenmodell eines Zeppelins, das im Viktoria-KinephonTheater zu bestaunen war. Aufgehängt wurde es während einer ersten Welle der AeronautikEuphorie um 1909, die sich in den Dresdner Kinos auch in mindestens vier Luftschiff-Filmen niederschlug. ${ }^{18}$

16 Teil C, ergänzend Teil D, der nur sechs Blatt umfasst.

17 Sammlung Ott, Teil C, Blatt 50.

18 Siehe Sammlung Ott, Teil M: Chronologisches Verzeichnis aller Filme von 1906 bis 1936, Blatt 8-18 für die Jahre 1908 und 1909.
Diese Streifen gehören zu jenen rund 6.500 Filmen, die in den Teilen $L$ und $M$ der Sammlung Ott sowohl in alphabetischer als auch in chronologischer Ordnung aufgelistet werden. Dabei erfolgt eine Verzahnung mit den Lichtspielhäusern, insofern jeweils das Dresdner Erstaufführungskino zugewiesen ist. Allerdings sind diese Listen nicht vollzählig, wie ein Abgleich mit dem Tagebuch von Victor Klemperer zeigt, in dem der begeisterte Dresdner Cineast über 15 Jahre hinweg, bis ihm als Juden seitens des nationalsozialistischen Staats am 12. November 1938 Kinobesuche verboten wurden, die angeschauten Filme kommentiert. ${ }^{19}$

Warum Otts Filmlisten erst 1936 enden, die Kinolisten aber schon im Jahr 1933, ist derzeit nicht zu erklären. Zumindest entstanden, wie die Dresdner Adressbücher beweisen, auch in den drei Jahren von 1934 bis 1936 mit dem Olympia und den Park-Lichtspielen Lichtspielhäuser in Dresden. Für den Zeitraum bis 1949, das den Schlusspunkt des Untersuchungszeitraumes des Dresdner Kinoprojektes markiert, konnten durch Abgleich mit weiteren Quellen insgesamt zehn, zum Teil nur temporäre Spielstätten ermittelt werden, die nicht bei Ott gelistet sind. Zu nennen sind etwa Filmaufführungen im Zirkus Sarrasani, dessen festes Zirkusgebäude in den 1920er-Jahren von anderen Veranstaltern gemietet werden konnte, oder in der Sächsischen Staatsoper, der Semperoper, in der am 10. Januar 1926, 15 Jahre nachdem dort „Der Rosenkavalier" Weltpremiere gefeiert hatte, die

19 Siehe Klemperer Online, vor allem das dort beigefügte chronologische Verzeichnis der von Victor Klemperer gesehenen Filme. 
Verfilmung der Strauß-Oper erstaufgeführt wurde. ${ }^{20}$ Die Kino- und Filmlisten bilden die beiden Hauptteile der Sammlung Ott. Die übrigen Teile bestehen aus Texten zum Beispiel über die Dresdner Filmverleiher, Die Gründung des Vereins der Dresdner Kinobetreiber oder über technische Aspekte, wie der Entwicklung des Tonfilms. ${ }^{21}$ Sie gewähren zusätzliche Einblicke in die zeitgenössische Kinowelt und deren Verankerung im urbanen Umfeld.

\section{Dresdner Kinokultur im Spiegel der Sammlung Ott}

\section{Dresden auf dem Weg zur Kinometropole}

Die ersten Einträge der Sammlung Ott führen auf die Dresdner Vogelwiese, das bekannteste Volksfest der sächsischen Residenz. Damit verweist die Quelle auf einen der beiden Wege, auf denen sich die neue Erfindung des Kinos mit großer Geschwindigkeit verbreitet hat. Dessen Geburtsstunde schlug Ende 1895, als unabhängig voneinander Max und Emil Skladanowsky in Berlin und die Gebrüder Lumière in Paris Filme vor zahlendem Publikum aufgeführt hatten. Bereits seit dem Folgejahr gastierten Wanderkinos

\footnotetext{
20 Zur Rosenkavalier-Verfilmung von 1925 in der Sächsischen Staatsoper siehe https://www.filmportal.de/ film/der-rosenkavalier_caa73c6fb94348819654781 9420d0739.

21 Teil B (Dresden und die Kinematographie), Teil E (bewegtes Bild), Teil F (Filmverleiher), Teil G (Filme einst), Teil H (Tonfilm), Teil J (Verein der Dresdner Kinobesitzer) und Teil K (Lustbarkeitssteuer).
}

auch in Dresden. ${ }^{22}$ Den Anfang machten die beiden Hamburger Schausteller Dienstknecht und Meyer, es folgten Unternehmen zum Beispiel aus Löbau und Leipzig, aber auch aus Pirmasens. Insgesamt gastierten 15 Wanderkinematographen zum Teil mehrfach in der Elbestadt, wo in manchen Jahren bis zu vier Betriebe gleichzeitig um die Gunst des Publikums warben.

Solche Wanderkinos entstanden, als Schaubudenbesitzer auf der ständigen Suche nach neuen Attraktionen begonnen hatten, ihre Sensationstheater umzurüsten. ${ }^{23}$ Dabei korrespondierte die äußere Gestalt dieser beweglichen Kinos mit der Sensation des neuen Mediums. Wie Heinrich Ott berichtet, hatten manche Betreiber sogar Oelmotore oder grosse Locomobile angeschafft, womit sie ihren elektrischen Strom erzeugten, der nicht nur zur Projektion, sondern auch zur Beleuchtung ihrer Prunkfassaden diente und so entstanden Prachtbauten [...], die mitunter einen Gesamtwert bis zu 80.000 Mark repräsentierten. ${ }^{24}$ Offenkundig erwiesen sich die Wanderkinos als hochprofitabel - nicht zuletzt, weil die laufenden Kosten gering waren. ${ }^{25}$ Die Ursache für diese Rentabilität lag wesentlich darin, dass das Wanderkino auf einfach gestaltete Filmprogramme setzen konnte, weil aufgrund der kurzen Dauer des Jahrmarktes dem ortsgebundenen Publikum nur ein kleines Zeitfenster zur Verfügung stand, um seine Sensationslust zu stillen. Eine Vorstellung, die höchstens

22 Zum Wanderkino siehe den Aufsatz von Sophie Döring in diesem Band sowie Müller/Döring: „Der Kino“, S. 71-79; vgl. insgesamt auch Flügel: Achsenjahr.

23 Siehe Klunkert: Schaustellungen, besonders S. 358369.

24 Sammlung Ott, Teil C, Blatt 3.

25 Garncarz: Öffentliche Räume, S. 38. 


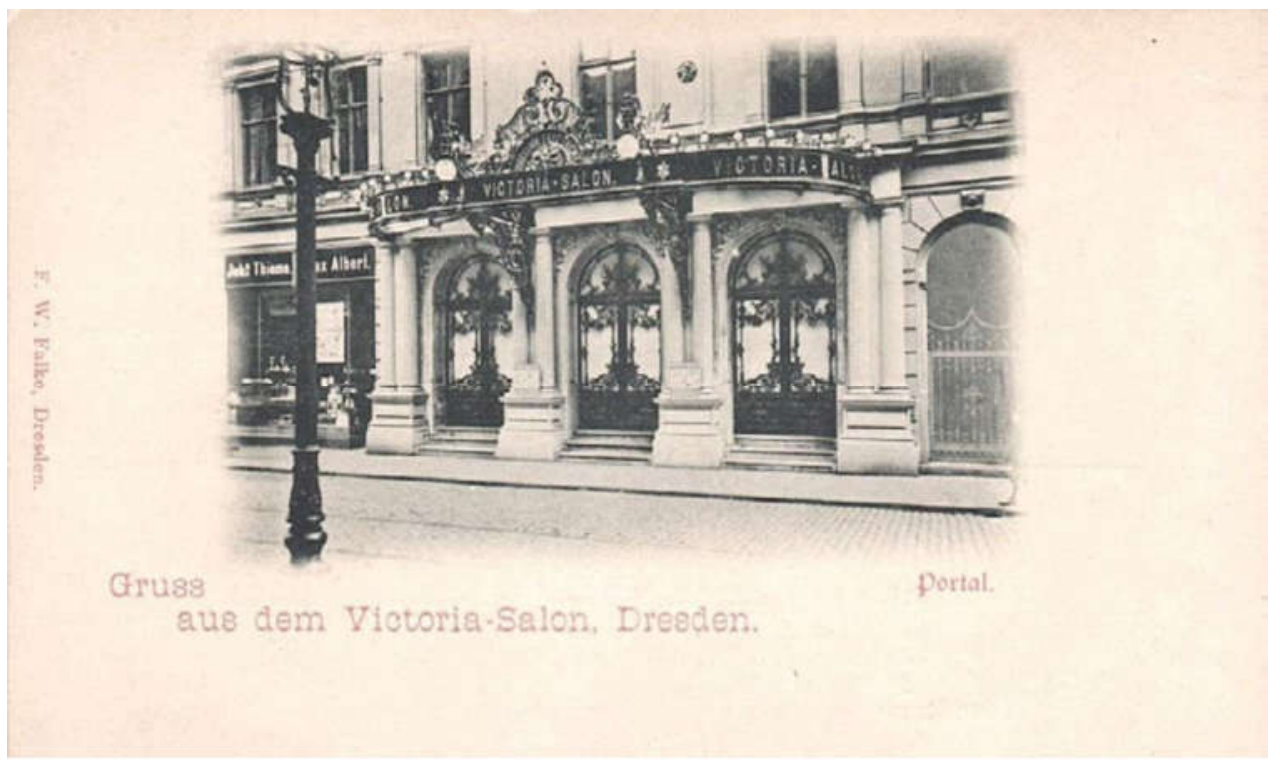

Abbildung 2: Victoria-Salon, Postkarte undatiert (Quelle: https://altesdresden.de/pics/seeo/wais0265.jpg).

25 Minuten dauerte, bestand aus etwa 18 bis 20 Kurzfilmen, zumeist Humoresken und sogenannten Tagesereignissen. Die einzelnen Streifen wurden von den Schaustellern gekauft und auf den Jahrmärkten der unterschiedlichen Orte in Endlosschleifen gezeigt, bis die Verschleißgrenze erreicht war. ${ }^{26}$

Neben dem Jahrmarktkino verweist die Sammlung Ott auf eine großstädtische Kulturinstitution, die, wenngleich in geringerem Maß als der Wanderkinematograph, ebenfalls zur Popularisierung des neuen Mediums beitrug: nämlich das Varietétheater. ${ }^{27}$ Beispielhaft belegen seit

26 Allgemein zur Programmentwicklung im frühen Kino siehe Müller: Kinematographie.

27 Siehe auch Müller: Kinematographie, S. 11.
Oktober 1903 Zeitungsinserate in der Dresdner Tagespresse, dass im Victoria-Salon und im Central-Theater Lebende Photographien als Abschlussnummer des Programms zur Vorführung kamen. ${ }^{28}$ Damit begannen die Varietés, für das eigene Geschäftsmodell ein Medium zu nutzen, dessen Erfolg sich seit 1896 verstetigt hatte. Hierbei kam innen einerseits die strukturelle Gleichheit der Programmgestaltung beider Institutionen entgegen, andererseits konnten sie mit ihrem Angebot in die große zeitliche Lücke stoßen, die zwischen den Besuchen der Wanderkinematographen in Dresden bestand. Unbeschadet dessen blieb die Vogelwiese mit ihren

28 Sammlung Ott, Teil C, Blatt 5. 
Wanderkinos, die ungleich mehr Leute anzogen als das Varieté, die jährliche Kinohauptsaison. ${ }^{29}$ Bis 1905 blieb diese Situation unverändert. Doch 1906 kam es zu einem überraschenden Wandel, als - nach einem außerordentlich frühzeitigen, von Ott nicht dokumentierten Fehlversuch einer Kinogründung im Jahr 1902 - nicht nur in Dresden, sondern auch in zahlreichen deutschen Großstädten eine Welle von Kinogründungen einsetzte. ${ }^{30}$ Es kam zu einer regelrechten ,Goldgräberstimmung', wie der Kommentar eines Kommissars der Dresdner Wohlfahrtspolizei illustriert: Die Kinematographen schiessen jetzt wie Pilze aus der Erde, und die Besitzer glauben, dass jeder Keller, jeder zufällig ungenutzte Lagerraum geeignet erscheint, darin Vorführungen zu veranstalten. ${ }^{31}$ Allein 1906 wurden in der sächsischen Metropole 15 Kinematographen gegründet. Für die Folgejahre bis 1911 verzeichnet Ott die Gründung von jährlich neun bis 15 Kinos, insgesamt waren es im genannten Zeitraum 75. Hinzu kamen verschiedene temporäre Aufführungsstätten, deren Betriebsdauer wie beim Reform-Kino auf der Hygiene-Ausstellung 1911 von Anfang an auf wenige Wochen begrenzt war. ${ }^{32}$ Doch die Gründung von Kinos rief nicht nur Euphorie hervor, sondern stieß zuweilen auch auf Widerspruch, was die ambivalenten

29 Zur im Vergleich zum Varieté größeren Anziehungskraft des Kinos siehe Haller: Kino, S. 234.

30 Zur Kinogründung von 1902 siehe Zeh: Lichtspieltheater, S. 182, basierend auf Sächsischer Bote,

1. Woche Januar 2003.

31 Sammlung Ott, Teil C, Blatt 23-24.

32 Gegründet wurden 1906: 15 Kinos; 1907: 11 Kinos; 1908: 15 Kinos; 1909: 10 Kinos; 1910: 9 Kinos; 1911: 15 Kinos; 1912: 6 Kinos; 1913: 2 Kinos. Einige dieser Kinos wie Das kleine Theater (1908) sind in der Sammlung Ott nicht enthalten.
Befindlichkeiten spiegelt, die das neue Medium Film hervorrief. Da Kinder und Jugendliche einen wichtigen Teil des Publikums ausmachten, musste der Besitzer des 1909 eröffneten Viktoria-Kinephon-Theaters, das sich im selben Gebäude wie eine Schule befand, seinen Kritikern und der Wohlfahrtspolizei zusichern, dass die Vorstellungen erst ab 16:00 Uhr, mithin nach Schulschluss, anfingen. Weitergehende Befürchtungen der Schulleitung und der Eltern aufgreifend, versprach er zudem: die so sehr verachtete Schundromanliteratur in Filmvorführungen [ist] in unserem Programm ausgeschlossen. ${ }^{33}$

Im Ergebnis des Gründungsbooms hatte sich das Kino als fester Bestandteil der Massenkultur in Dresden endgültig etabliert und war dabei den Kinderschuhen entwachsen. ${ }^{34}$ Dies bestätigt auch ein Blick in die lokalen Adressbücher. Seit der Ausgabe von 1911 enthält der Branchenteil eine eigene Rubrik Kinematographen, zuvor waren die Lichtspiele nur im Straßen- beziehungsweise über ihre Besitzer im Einwohnerverzeichnis zu finden gewesen. Zudem hatte das Jahrmarktskino bereits im Vorjahr vor der massenhaft auftretenden ortsfesten Konkurrenz kapituliert. Mit der Dresdner Vogelwiese 1910 endete in der Elbmetropole die Ära des Wanderkinematographen, der sich jedoch in kleineren Städten noch weiterer Beliebtheit erfreute. Ebenso stellte das Dresdner Gewerbeamt in den wirtschaftlich schwierigen Jahren nach dem Ersten Weltkrieg bis in die Mitte der 1920er-Jahre hinein Konzessionen für mobile Kinematographen aus, die jedoch den Glanz der mobilen Kinopaläste der Vorkriegszeit missen ließen. Anstatt

33 Sammlung Ott, Teil C, Blatt 65-66.

34 Jubiläums-Festschrift, S. 7. 
einer Lokomobile und eines aufwendigen Kinopalastes reichte dem in Dresden wohnhaften Schausteller Bruno Herklotz ein einspänniges Pferdefuhrwerk zum Transport des Wanderkinematographen, den er vermutlich nicht in einem eigenen Zelt, sondern in angemieteten Sälen aufbaute. ${ }^{35}$

\section{Voraussetzungen des Kinobooms}

Der urbane Kinoboom fußte auf zwei Voraussetzungen. Erstens, wie Joseph Garncarz argumentiert, auf dem Erfolg des Wanderkinos, der zu einer stetig wachsenden Filmnachfrage führte. Im Ergebnis entstand eine Filmindustrie, die ab 1905 jene Quantität an Filmen produzierte, die für einen regelmäßigen Programmwechsel notwendig war, um den gewachsenen Ansprüchen des großstädtischen Publikums zu genügen und es immer wieder ins Kino zu locken. ${ }^{36}$ Diese Beobachtung spiegelt auch die Sammlung Ott, insofern deren Filmliste erst mit dem Jahr 1906 einsetzt. In Verbindung mit der aufstrebenden Filmindustrie hat sich zudem ein Filmmarkt und der Filmverleih als neuer Vertriebsweg herausgebildet, so dass nun die Kinos permanent und preisgünstig mit neuen Streifen versorgt werden konnten. In Dresden gab es bereits seit 1906 verschiedene Kinobesitzer, die sich zugleich als professionelle Filmverleiher betätigten und in der Stadt selbst entwickelte sich 1910 die Urzelle des Film-Verleih-Verbandes. ${ }^{37}$ Zu hinterfragen ist, ob in kausaler Verknüpfung mit diesen Entwicklungen auch die Kinotechnik

35 Stadtarchiv Dresden, Gewerbeamt 2.3.9. _ 5507, Film-Nr. H0211, Bruno Herklotz, 1923 und 1924.

36 So explizit Garncarz: Räume, S. 38; siehe ebenso Garncarz: Entstehung, S. 151.

37 Sammlung Ott, Teil F: Dresdner Filmverleiher, Blatt 3. erschwinglicher wurde, was der Einrichtung von Kinematographen weiteren Vorschub geleistet hätte. Hierzu stehen Untersuchungen aus, aber bereits der Umstand, dass der Dresdner Unternehmer Heinrich Ernemann spätestens seit 1904 Kameras und seit 1911 auch Filmprojektoren jeweils für den Heimbedarf produzierte, ist als Indiz für einen Preisrutsch bei der Kinotechnik zu verstehen. ${ }^{38}$

Zweitens kann mit Blick auf den soziokulturellen Kontext festgehalten werden, dass das Kino keineswegs zufällig in den Metropolen, den "Experimentierfelder[n] der Vergnügungskultur", sesshaft wurde. ${ }^{39}$ Denn nur in Großstädten wie Dresden, das 1910 mit seinen 548.308 Einwohnern fünftgrößte Stadt im Kaiserreich war, fand es die Voraussetzungen für seinen Aufstieg zum Leitmedium einer neuen Massenkultur. Einerseits, so argumentiert Kaspar Maase, existierte hier ein ausreichend großes Publikum mit neuen Freizeiterwartungen, die vom Takt des industriellen Zeitalters, vom urbanen Leben und moderner Lohnarbeit geprägt waren. ${ }^{40}$ Andererseits bestand in den Großstädten ein Angebot kommerzieller Populärkünste, das dank steigender Einkommen für breite Schichten erschwinglich war. Gewinnorientiert zielte es darauf ab, dem starken Unterhaltungsbedürfnis des Publikums zu genügen. Das entscheidend Neue im frühen 20. Jahrhundert lag nach Maase darin, dass diese Angebote dank neuer Techniken für große

38 Siehe Vincenz/Hesse: Fotoindustrie, Katalogteil S. 265 und 275.

39 Maase: Vergnügen, S. 66.

40 Maase: Vergnügen, S. 20-21. Hier auch das Folgende. 
Bevölkerungskreise alltäglich - eben Massenkultur - wurden. ${ }^{41}$

Hier schließt das als „städtisches Erlebnismodell" begriffene Kino unmittelbar an. ${ }^{42}$ In Marktmechanismen eingebunden, nutzte es die urbanen Infrastrukturen und bedingte ebenso die Entwicklung neuer Industrien, wie es als Arbeitsplatz neue Berufsgruppen schuf. ${ }^{43}$ Die Kausalität dieser Entwicklung verdeutlicht der Blick auf die Ernemann-Werke, die einen wesentlichen Beitrag an Dresdens Aufstieg zu einem Zentrum der Filmtechnik hatten. Einerseits reagierten sie mit ihren Projektoren auf die wachsende Nachfrage der Kinobranche, andererseits schulten sie die zur Bedienung der technisch immer aufwendigeren Kinomaschinen notwendigen Filmvorführer. ${ }^{44}$ Zugleich passte sich das Lichtspielwesen der durch die Hochindustrialisierung normierten Teilung von Arbeit und Freizeit an. Ein Spezifikum des Kinos, das Corinna Müller und Harro Segeberg betonen, bestand in seiner Mittlerfunktion zwischen den traditionellen Medien der Unterhaltungskultur und der neuen Massenkultur. ${ }^{45} \mathrm{Als}$ öffentlicher Ort des Sehens und Gesehenwerdens glich es einerseits traditionellen Formen, etwa der Musikbühne oder den Varietés. Andererseits bildete es einen sogenannten dritten Ort, das heißt einen Lebensraum, der zwischen dem eigenen Zuhause und

41 Neben Maase siehe hierzu auch Wietschorke: Arbeitsgemeinschaft, S. 16-20.

42 Gottfried Korff, zitiert nach Haller: Kino, S. 229.

43 Zu den neu entstandenen Berufsgruppen im Kinogewerbe siehe den Aufsatz von Merve Lühr in diesem Band.

44 Zu Dresden als Zentrum der Kinotechnik siehe Forster: Kino, S. 3-12, zur Schulung der Filmvorführer siehe Jubiläums-Festschrift, S. 14-15.

45 Müller/Segeberg: Öffentlichkeit, S. 15. dem Arbeitsplatz angesiedelt ist. ${ }^{46}$ Ohne Zwang auf Verhaltensnormen auszuüben, wirkte es einladend und stand aufgrund niedriger sozialer Zugangsbarrieren allen Schichten der Bevölkerung offen: Heinrich Ott vermerkte in seiner Quelle, dass das im Juli 1906 gegründete Dedrophon mit seinen geringen Eintrittspreisen auf untere soziale Schichten zielte, nichtsdestotrotz aber am 31. Dezember 1906 vom sächsischen König und seinen Kindern besucht worden war. ${ }^{47}$

\section{Kinoentwicklung in Zahlen}

Mit Ende dieses frühen Kinobooms war die Ausbreitung des Kinos natürlich noch längst nicht gestoppt. Lediglich eine erste Marktsättigung war eingetreten, so dass sich nach 1911 die Zahl der jährlichen Kinoneugründungen auf zwei bis fünf einpendelte und mit wenigen Ausnahmen - etwa in den Jahren des Ersten Weltkriegs und insbesondere der Inflation, die dem Kino schwer zu schaffen machten ${ }^{48}$ - bis in die Jahre um 1930 auf diesem Niveau verharrte. Allerdings schlossen viele Kinematographen nach kurzer Zeit ihre Pforten wieder, womit die Zahl der Kinos, die jeweils zeitgleich in Betrieb waren, in Dresden bei jährlich etwa 35 bis 40 lag. Die absolute Spitze markiert das Jahr 1911 mit 45 Kinos, im Ergebnis der Kriegsfolgen und der Hyperinflation sank die Zahl 1924 auf etwa 30, um bis zu Beginn des Zweiten Weltkrieges auf 36 zu steigen. Doch während der Mittelwert stabil blieb, verdreifachte sich die Zahl der Kinositzplätze von etwa 7.000 im Jahr 1914 über etwa

46 Zum Begriff siehe Oldenburg: Place.

47 Zum Thema Monarch und Kino siehe den Aufsatz von Winfried Müller in diesem Band.

48 Siehe Jubiläums-Festschrift, S. 12 und 15. 
11.300 und 19.300 in den Jahren 1924 und 1928 auf $22.194 \mathrm{im}$ Jahr 1932. Mit anderen Worten: Zu Beginn des Ersten Weltkrieges standen pro 1.000 Einwohner knapp 13 Kinoplätze zur Verfügung, im letzten Jahr der Weimarer Republik waren es mit 34 mehr als doppelt so viele. ${ }^{49}$ Der Erste Weltkrieg markierte übrigens für das Kino als Phänomen der Massenkultur keinen gravierenden Einschnitt. Nach den ersten Kriegswochen hatte sich das Dresdner Kulturleben unter den veränderten Bedingungen rasch wieder normalisiert. Insbesondere das urbane Kino verwies auf eine blühende Vergnügungskultur, die eine willkommene Ablenkung bot. Eine Steigerung erfuhr die Kinobegeisterung schließlich im letzten Kriegsjahr. Nachdem mit dem Steckrübenwinter 1916/17 der Krieg endgültig auch in der Heimat angekommen war, entdeckte man im Deutschen Reich die Propagandakraft der Filme, die so zum Objekt staatlicher Kontrolle und Förderung wurden. Anfang des Jahres 1917 wurde auf Veranlassung der Obersten Heeresleitung das Bild- und Filmamt (Bufa) mit dem Ziel gegründet, die Verwendung von Filmmaterial mit militärischen Inhalten zu koordinieren. Hierbei entstanden Streifen wie „Der magische Gürtel", der in scharfem Kontrast zur Wirklichkeit des uneingeschränkten U-Boot-Krieges in propagandistischer Absicht eine geradezu ritterliche Kriegsführung der kaiserlichen U-Boot-Flotte vor Augen führte. Im Dezember 1917 folgte schließlich unter Beteiligung der Reichsregierung die

49 Zum Vergleich: 2008 waren es 12.000 Plätze, siehe https://www.dresden.de/media/pdf/statistik/Dresdner_Zahlen_aktuell_16_2009_08.pdf. Damit kamen auf 1.000 Einwohner gut 23 Kinositzplätze. Zu den Sitzplätzen vergleiche auch den Beitrag von Carola Zeh in diesem Band.
Gründung der Universum-Film AG (Ufa) mit dem Auftrag, Spiel- und Dokumentarfilme sowie Wochenschauen zu produzieren. Damit war ein Kinokonzern entstanden, dessen Filme in den folgenden Jahren die Kinosäle füllten und der selbst in den vielen Großstädten, so auch in Dresden, Lichtspielhäuser besaß.

Den auch während des Ersten Weltkrieges anhaltenden Aufschwung des Kinos widerspiegeln die Aussagen der Sammlung Ott zu den Filmprogrammen, in denen der seit 1910 bemerkbare abendfüllende narrative Spielfilm binnen weniger Jahre die alten, aus Kurzfilmen zusammengestellten Abfolgen verdrängte. ${ }^{50}$ Auch wenn die Programmabfolgen und die regelmäßigen Programmwechsel aus unbekannten Gründen nicht für alle existierenden Kinos dokumentiert sind, so ist doch eine deutliche Tendenz erkennbar. Für das Jahr 1913 listet Ott mehr als 160 dieser Filmprogramme in 16 Lichtspielhäusern auf, für das letzte Kriegsjahr nennt er mehr als 250 in zehn Kinematographen! Dieser Befund korrespondiert mit den Kinozahlen: 1914 gab es in Dresden 44 Kinematographen, im letzten Kriegsjahr waren es $41 .^{51}$

Allerdings täuscht die relative Konstanz der absoluten Kinozahl - die Kinobranche war nämlich von einer hohen Fluktuation gekennzeichnet: Allein von den 75 Kinos, die in den Boom-Jahren 1906 bis 1911 den Spielbetrieb aufnahmen, überlebten 25 (darunter acht von den 14, die 1906 gegründet worden waren) die ersten zwölf Monate nicht. Falls dieser Zeitraum überstanden

\footnotetext{
50 Siehe Haller: Kino, S. 250 und Müller: Kinematographie, S. 186-190.

51 So auch mit Rückgriff auf die Sammlung Ott Zeh: Lichtspieltheater, Liste der Kinostandorte, S. 182-192.
} 
war, betrug die durchschnittliche Betriebsdauer knapp zehn Jahre, wobei eines der ältesten Dresdner Kinos, das Dedrophon, bis zur deutschlandweit verfügten Schließung aller Kinos am 4. Februar 194539 Jahre lang in Betrieb war. Insgesamt stieg die durchschnittliche Betriebsdauer insbesondere bei jenen Kinos an, die nach dem Ersten Weltkrieg gegründet wurden, was mit einer zunehmenden Professionalisierung und Technisierung zu erklären ist.

Eine ähnliche Tendenz zeigt ein Blick auf die häufigen Besitzerwechsel. Im Durchschnitt änderten sich die Besitzverhältnisse der Dresdner Kinos alle 4,2 Jahre - im Umfeld der Metropole, das mit seinen kleinen Städten und geringeren Publikumszahlen die Kinobetreiber offenbar vor größere Herausforderungen stellte als die Großstadt, wechselten die Kinos sogar alle zwei bis drei Jahre den Eigentümer. ${ }^{52}$ Die beiden Pole in der Elbestadt bilden einerseits das 1911 gegründete Volkskino, das während seiner elfjährigen Existenz 18 verschiedene Eigentümer hatte, und die 1927 errichtete Schauburg. Sie blieb über 18 Jahre hinweg in der Hand von Arnulf Huyras, einem gebürtigen Genfer, der ab 1914 in Leipzig lebte und hier nach dem Ende des Ersten Weltkrieg als Leiter der Mitteldeutschen UniversumFilm-AG-Verleihbetriebe tätig war. ${ }^{53}$

Über die jeweiligen Gründe einer Geschäftsaufgabe beziehungsweise eines Besitzerwechsels schweigt Ott im Regelfall, allerdings erlauben mitunter die Akten des Gewerbeamtes weitergehende Aussagen. Danach musste im Jahr 1910

52 Die Angabe für den Dresdner Bezirk bei JubiläumsFestschrift, S. 18.

53 Siehe https://www.neustadt-ticker.de/57108/aktuell/ schauburg-grundungsurkunde-gefunden. das Vitascope-Theater schließen, weil nur das in unmittelbarer Nachbarschaft neu eröffnete Colosseum die Lizenz für einen Bierausschank und damit einen Wettbewerbsvorteil erhielt, den sein Konkurrent nicht ausgleichen konnte. ${ }^{54}$ In Einzelfällen ist aber auch zu fragen, ob tatsächlich ein dauerhafter Kinobetrieb angestrebt war. Wenn, wie die Sammlung Ott berichtet, der Gastwirt Alfred Haufe den Filmprojektor außerhalb des Gebäudes aufstellte, um die Filme durch ein Fenster in den Gastraum zu projizieren, dann klingt dies sehr nach einer auf wenige Tage terminierten Vorführaktion. ${ }^{55}$

Der Besitzwechsel selbst zählte offenkundig zu den normalen Vorgängen im Geschäftsalltag. Darauf deutet zunächst der Umstand, dass weder die hohe Fluktuation der Besitzer noch die teilweise kurze Betriebsdauer Alleinstellungsmerkmale der Kinobranche waren. Vielmehr zeigt der Abgleich der in der Sammlung Ott hinterlegten Kinoadressen mit den Dresdner Adressbüchern von 1905 bis 1940, dass zahlreiche Ladenlokale - nicht nur solche, die sich in den selben Gebäuden wie die Kinos befanden - ihren Inhaber und in Verbindung damit das Gewerbe häufig wechselten. Weiterhin kündet die Fluktuation nicht nur von einer Vitalität der Kinobranche und zumindest in den ersten Jahren von einer Goldgräberstimmung, sondern steht auch in kausalem Zusammenhang mit einem regelrechten Kinomarkt, von dessen Existenz zahlreiche Zeitungsannoncen künden. Eine Stichprobe in der lokalen Tagespresse ergab, dass die Dresdner Neuesten Nachrichten etwa

54 Stadtarchiv Dresden, Gewerbeamt, 2.3.9. - Film H7238, Hermann Hirschberg, 1908-1910.

55 Siehe Sammlung Ott, Teil C, Blatt 33. 
am 19. August 1906 sowie am 10. Februar und am 17. April 1907 Kinokaufgesuche publizierten, ebenso am 3. Oktober 1908 ein Kapitalgesuch für einen Kinokauf. Interessant in diesem Kontext ist schließlich die Annonce vom 23. Januar 1915: erstklass[ige] Kinos in Dresden Umstände halber preiswert zu verk[aufen], nachzufragen bei Heinrich Ott, der hier offenkundig als Vermittler auftrat. ${ }^{56}$ Ergänzend dazu gab es in Fachzeitschriften Tipps, was man beim Kauf eines Kinos in rechtlicher oder wirtschaftlicher Hinsicht zu beachten habe. ${ }^{57}$

\section{Standortfaktoren und Kinoausbreitung}

Die skizzierten Entwicklungen verdeutlichen eine wechselseitige Bedingtheit von Kino und Massenpublikum. Daraus ergeben sich Folgen für die Wahl der Standorte. Beleuchtet man die Kinoadressen in der Sammlung Ott, dann zeigt sich, dass die Standorte idealiter zwei Hauptkriterien erfüllen sollten. Erstens musste eine gute Erreichbarkeit gewährleistet sein, und zweitens wirkte es sich positiv aus, wenn sich bereits Lokalitäten, die einen Besucherstrom anzogen, in der Nähe des geplanten Kinos befanden, so dass Mitnahmeeffekte erzielt werden konnten. Pars pro toto ist auf das 1906 gegründete Edison-Theater zu verweisen, das in der Nähe des Dresdner Hauptbahnhofs in unmittelbarer Nachbarschaft zu verschiedenen Hotels lag, oder auf Kinos, die unmittelbar in Hotels eingerichtet wurden. Ebenso müssen Lokalitäten erwähnt werden, in denen, wie im Konzerthaus

56 Dresdner Neueste Nachrichten vom 23.1.1915. Ich bedanke mich bei Sophie Döring und Lennart Krantz für die Recherche.

57 Siehe etwa Frank: Kauf oder Stäubli: Kino.
Stadt München, Filme und Orchestermusik unter einem Dach aufgeführt wurden, und auch eine Kneipe in der Nachbarschaft konnte für einen steten Publikumsstrom sorgen.

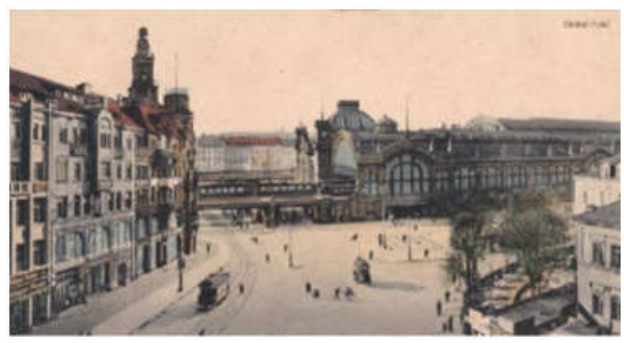

Abbildung 3: Die Edison-Lichtspiele neben dem Hotel New York in der Prager Straße, Postkarte 1913 (Quelle: https://altesdresden.de/pics/seeo/prag0471.jpg).

Die Kinoausbreitung im Stadtraum folgte letztendlich einem Muster, das sich ähnlich auch in anderen Großstädten erkennen lässt. In der sächsischen Elbmetropole verortet Ott die ersten festen Kinos im Stadtzentrum, allerdings zunächst nicht an den Hauptverkehrsadern mit ihren teuren Mieten, sondern in den Seitengäßchen der Hauptstraßen im Bereich der inneren Altstadt rund um das Residenzschloss und den Altmarkt. ${ }^{8}$ Hier ist auf das schon erwähnte Dedrophon zu verweisen, dass sich in der Wettiner Straße, der heutigen Schweriner Straße, und damit etwa 400 Meter vom Zwinger und vom Schauspielhaus entfernt befand. Als Kinostandort weiterhin beliebt waren die unmittelbar ans Zentrum angrenzenden Stadtteile sowie die vom Zentrum zum Hauptbahnhof führende Prager Straße. Von dieser Innenstadtrandlage aus

58 Jubiläums-Festschrift, S. 7. 
verdichtete sich das Kinonetz zunächst in die Mitte hinein. Schon Ende 1906 eroberte das Kino den Altmarkt sowie die zentralen innerstädtischen Verkehrsachsen, etwa die Wilsdruffer Straße und die Waisenhausstraße, wobei die letztere zu jenen Straßenzügen gehörte, welche die an der Elbe liegende Altstadt halbkreisförmig umschlossen. Dieses Areal blieb in den Folgejahren räumlicher Mittelpunkt der Dresdner Kinoszene; immerhin befanden sich hier 12 der 36 Lichtspielhäuser, die zu Beginn des Zweiten Weltkrieges existierten.

Eine zweite Stoßrichtung zeichnet sich ab, als die Kinos schon vor Ausbruch des Ersten Weltkrieges begannen, sich strahlenförmig in Richtung Peripherie auszubreiten. Regelrechte Kino-Cluster entstanden an den Hauptverkehrsachsen in der Dresdner Neustadt nördlich der Elbe, oder in den westlichen Arbeiterbezirken Trachenberge, Löbtau und Pieschen. Ebenso siedelten sich Lichtspielhäuser entlang eines Streifens an, der sich von der Altstadt nach Osten über die bürgerlichen Stadtteile Johannstadt und Striesen bis hin zur Elbbrücke in Blasewitz, dem sogenannten Blauen Wunder, zog. In den Jahren um 1930 erhielten schließlich auch einige der seit der Jahrhundertwende eingemeindeten Vororte wie Briesnitz, Cotta, Reick oder das gegenüber von Schloss Pillnitz an der Elbe gelegene Kleinzschachwitz eigene Kinos.

Mit dieser Eroberung des Stadtraums durch den Kinematographen relativierte sich die Frage nach der Entfernung zum Stadtzentrum. Erstens zielten diese Kinos insbesondere in Randlagen ausweislich ihrer Eröffnungsannoncen oft auf das unmittelbar lokale Publikum, andere wie das Vereinshaus Ost - heute das Programmkino Ost - zielten als Lokal der SPD auf einen bestimmten Personenkreis. Zweitens konnte der Distanz zum Zentrum mit dem Hinweis auf die Straßenbahnanbindung begegnet werden. So warb etwa Ott im Jahr 1926 in der Eröffnungsannonce für sein rund vier Kilometer vom Zwinger entfernt liegendes Theater am Bischofsplatz, es könne bequem mit zwei Straßenbahnlinien erreicht werden - das Kino nutzte also die urbane Infrastruktur ${ }^{159}$ Ebenso ist in diesem Kontext auf den Umstand zu verweisen, dass verschiedene Ausflugslokale am Stadtrand, die wie der Heidepark nun an das Straßenbahnnetz angeschlossen wurden, Kinos eröffneten.

\section{Das Erscheinungsbild des frühen Kinos}

Die explosionsartige Ausbreitung des Kinematographen und die hohe Fluktuationsrate der Kinobesitzer und -betreiber gerade in den frühen Jahren seiner Sesshaftwerdung legen nahe, dass die Hürden, ein Kino zu eröffnen, relativ gering waren. Damit korrespondiert, dass laut Sammlung Ott oftmals nur wenige Tage zwischen der Antragstellung und der Kinoabnahme durch die Behörden vergingen. Tatsächlich waren die Anforderungen zunächst nur gering, wie der folgende Auszug aus der Bekanntmachung der Dresdner Polizei vom 8. Mai 1909 verdeutlicht: ${ }^{60}$ Namentlich müssen a. sämtliche Türen nach außen schlagen, b. die Ausgangstüren mit der deutlich lesbaren Aufschrift ,Ausgang' versehen sein und während der Vorstellungen stets unverschlossen gehalten werden, c. Notlampen in der erforderlichen Zahl vorhanden sein und von Beginn der Vorstellungen ab bis zu deren Schlusse brennend erhalten werden,

59 Siehe Sammlung Ott, Teil C, Blatt 149.

60 Bekanntmachung, Paragraf 2. 
d. im Zuschauerraum genügend breite Gänge vorhanden und in der Regel die Stühle mit dem Fußboden oder unter sich durch Latten fest verbunden sein.

Diese Minimalanforderungen erfüllend, besaßen die Kinematographen oft nur die allernötigste Ausstattung - als Sitzmöbel diente im 1908 eröffneten Schloß-Salon ausrangiertes Kirchengestühl - und fanden ihren Platz in leerstehenden und deshalb leicht anzumietenden Ladenlokalen und ähnlichen Räumlichkeiten, die gerade zur Verfügung standen. Deshalb hat sich für diese Kinos seit 1912 der Begriff Ladenkino eingebürgert. ${ }^{61}$ Bis in die 1920er-Jahre hinein entstanden rund 40 solcher Ladenkinos, zu denen auch das Dedrophon zählte.

Die Gegebenheiten ausnutzend, entwickelte der sesshaft gewordene Kinematograph innerhalb kürzester Zeit eine große Formenvielfalt. ${ }^{62}$ Sie „erschwert es, eine einfache Typisierung vorzunehmen. Als nahezu unmöglich erscheint es, für alle vorkommenden Typen Begriffe festzulegen." ${ }^{33}$ Immerhin lassen sich hinsichtlich der baulichen Erscheinung grob vereinfacht drei Idealtypen benennen, die im Bearbeitungszeitraum nebeneinander existierten: Neben dem Ladenkino verortet die Forschung das sogenannte Saalkino, wobei die Anzahl der Sitzplätze als ein in der Literatur oft gebrauchtes Differenzkriterium nicht plausibel erscheint. Vielmehr ist Rolf-Peter Baacke zu folgen, der das entscheidende Charakteristikum der "Lichtspielsäle" darin sieht, dass sie Ball- oder Konzertsäle sowie

61 Siehe Müller: Kinematographie, S. 30 und S. 264, Anm. 15.

62 Siehe den Aufsatz von Carola Zeh in diesem Band, ebenso Neumann: Schaubude, S. 26-30.

63 Brosch: Kinobauten, S. 141. aufgegebene Kaufhäuser und Markthallen, und damit Räumlichkeiten nutzten, die für größere Menschenansammlungen konzipiert waren, umwidmeten und hier einiges an Luxus und technischen Neuerungen aufboten. ${ }^{64}$ Ein sehr frühes Beispiel für ein solches Saalkino ist das Campaneta, das Ott Ende 1906 in einem leerstehenden Kaufhaus nach dem ihm vertrauten Vorbild eines Theaters einrichtete, indem er im Interesse perfekter Sichtverhältnisse ansteigende Sitzreihen einbaute. Ähnlich agierten die Betreiber des Tonbild-Theater Olympia am Altmarkt, das 1909 dergestalt in ein ehemaliges Kaufhaus integriert wurde, dass die Galerien, die im zweiten Stockwerk den Hauptverkaufsraum umliefen, gleich einem Theaterrang als zusätzlicher Zuschauerraum dienten.

Mit dem Rückgriff auf das Theater zeichnet sich bereits eine Entwicklung ab, die zum dritten Idealtyp, den eigenständigen Kinoneubauten oder auch Kinopalästen führt. Deren Entstehung fällt nicht zufällig in den bereits erwähnten Zeitraum, zu dem der abendfüllende narrative Spielfilm entstand und im Zusammenhang damit der Film in eine Phase wirtschaftlicher und künstlerischer Konsolidierung eintrat. Vor diesem Hintergrund hatte die Zeitschrift Der gute Geschmack bereits 1911 gemahnt, schließlich muß eine so volkstümliche Erscheinung [das Kino - Anmerkung des Verfassers] doch irgendwie einmal münden vor den Händen und Sinnen eines schöpferischen Architekten, der aus

64 Siehe Baacke: Lichtspielhausarchitektur, S. 8-9. Zur Umwandlung von Ballsälen siehe auch Kukula/Helas: Ballhäuser, S. 163, 168-171, 173, 191. Im Unterschied dazu das temporäre Saalkino, vergleiche den Aufsatz von Sophie Döring in diesem Band. 
der besonderen Geste dieser Bilderspiele seine Raumdisposition entwickelte. ${ }^{65}$

Diese Entwicklung wiederspiegeln die nun entstehenden Kinobauten, die aus dem „Schatten der Improvisiertheit und Behelfsmäßigkeit" der früheren Kinos heraustraten und zur "vitalsten und ambitioniertesten" architektonischen Aufgabe der 1910er- und 1920er-Jahre wurden. ${ }^{66}$ Dresden erhielt schon frühzeitig, noch vor Ausbruch des Ersten Weltkriegs, gleich zwei dieser neuen Kinos. Ihre Architekten meisterten die gestellte Herausforderung, repräsentative, den Ansprüchen des Massenpublikums genügende Säle in hochverdichteten Stadträumen zu platzieren, auf unterschiedliche Weise. Zum einen errichtete Martin Pietzsch das 1911 eröffnete Union-Theater (U.T.) als Neubau in einem Hinterhof, wobei eine Passage die Verbindung zur Eingangshalle herstellte, die sich ihrerseits in einem Gebäude an der Straßenfront befand. Zum anderen erbaute Adam Gutjahr im Folgejahr das Rodera, indem er den Zuschauersaal mit großen Eingriffen in die Innenarchitektur bestehender Wohn- und Geschäftshäuser - heute würde man sagen: durch Entkernung - integrierte.

Mit der bereits erwähnten Gründung der Ufa im Dezember 1917 begann mit der Ära der professionalisierten Produktion von Großfilmen auch in Deutschland ein Prozess, der in den 1920erJahren zur Errichtung zahlreicher weiterer Kinopaläste führte - in Dresden auch unter Rückgriff auf die in U.T. und Rodera vorgeprägten Lösungen. Hinzu kamen Saalanbauten an bestehende Gebäudekomplexe, etwa beim Faun-Palast. Doch während in Berlin 1913 mit dem Cines am
Nollendorfplatz der erste freistehende Kinoneubau in Deutschland entstand, fand diese Bauform erst 1926 mit der Schauburg Eingang nach Dresden. ${ }^{67}$ Verantwortlich für diese Verspätung war jedoch nicht die mangelnde Phantasie der in der Elbestadt tätigen Architekten, sondern schlicht die lokale Bebauungssituation.

Die skizzierte Ausprägung von Laden-, Saal- und eigenständigen Kinos dokumentiert eine formale Diversität, die mit technischen und kulturellen Entwicklungen korrespondiert. Zunächst war die Entstehung der neuen großen Lichtspieltheater unmittelbar mit Fortschritten in der Kinotechnik verbunden. Für Zuschauersäle, die wie der des U.T. 1.000 Besuchern Platz boten, mussten die Filmprojektoren anstelle des anfänglich weit verbreiteten, schwachen Kalklichts starke, das heißt elektrische Projektionslampen erhalten. Weil dadurch die Brandgefahr anstieg, wurden zugleich in den Kinos neue Sicherheitsvorrichtungen wie baulich eigenständige Vorführkabinen durchgesetzt. Zugleich verweist die Ausprägung der drei Kinotypen auf zwei kulturelle Aspekte. Erstens belegt der Umstand, dass zwischen 1906 und den späten 1920er-Jahren in Dresden um die 30 Ballsäle zu Kinos umgewidmet wurden, eine Verschiebung innerhalb der Freizeitkultur. Zweitens transportiert die Innenarchitektur von Lichtspielhäusern wie dem U.T. eine symbolische Aussage. Indem schon vor dem Ersten Weltkrieg klassische, bislang dem Theater vorbehaltene Gestaltungselemente wie Ränge, Logen, ansteigende Sitzreihen und ausgefeilte Beleuchtungsanlagen übernommen

67 Eine zeitgenössische Würdigung des Cines in Kinotheater-Bau.
65 Zitiert nach Vaupel: Sperrsitz, S. 2.

66 Slidatke: Rummelplatz, S. 3 und 15. 


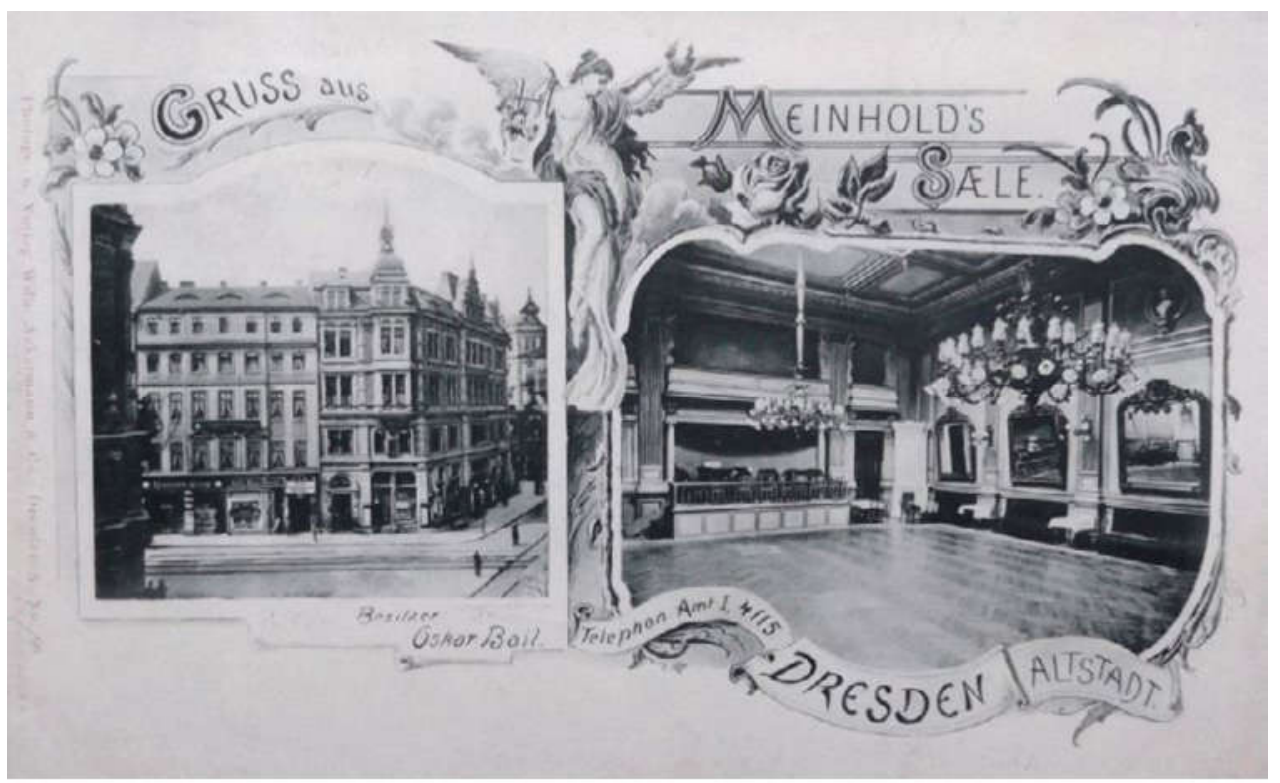

Abbildung 4: Das Ballhaus Meinholds Säle, die späteren M.S.-Lichtspiele, Postkarte 1901 (Quelle: https://altesdresden.de/pics/alts/mori0101.jpg).

wurden, demonstrierten die Kinos ihren Anspruch auf kulturelle Ebenbürtigkeit.

Mit der Innenarchitektur korrespondieren das äußere Erscheinungsbild des modernen Kinobaus und seine Prägekraft im Straßenbild. Die zuweilen mit Papier zugeklebten Fenster, mit denen in den frühen Ladenkinos die notwendige Dunkelheit im Zuschauersaal erreicht wurde, und die wohl in der Tradition der mobilen Kinopaläste stehende Fassadenbemalung des Dedrophon waren überwunden. Stattdessen fanden zunehmend illustrierte Filmplakate Verwendung. Musste im April 1915 der Betreiber der M.S.-Lichtspiele die Behörden noch um Erlaubnis bitten, um drei dieser Plakate, die eine ganz besondere Wirkung besäßen, am Kinoeingang anzubringen, so erhielten wenige Jahre später Lichtspielhäuser wie die Schauburg effektvolle, fensterlose Fassaden, die viel Platz für diese neue Form der Außenwerbung ließen. ${ }^{68}$ Hier zeigt sich eine Modernität, die noch einmal überboten werden sollte durch die Lichtreklame der Kinopaläste, die nach der Aufhebung der behördlichen Einschränkungen des Stromverbrauchs ab 1924 als Höhepunkt der städtischen Beleuchtung mit elektrischem Licht galten und damit zum Symbol für urbanen

68 Zu den M.S. Lichtspielen siehe Sammlung Ott, Teil C, Blatt 149. 
Fortschritt schlechthin wurden. ${ }^{69}$ Mit diesen Palästen hat sich der Film in die Innenstädte und damit auch in das Bewusstsein seines Publikums hineingebaut. ${ }^{70}$

Einen speziellen Teil der Selbstdarstellung des Lichtspielhauses bildete schließlich der Name des Kinos. Die Namenswahl unterlag einem gewissen Kanon und erfolgte oft unter Gesichtspunkten des Marketings, wobei durchaus Mondänität gewünscht war. Es finden sich zum Beispiel royale oder patriotische Namen wie Prinzess, Wettin-Kino, Deutscher Kaiser, Dreikaiser-Lichtspiele sowie Germania und Vaterland. Dies kann ein Ausdruck des Zeitgeistes sein, aber ebenso wurden bereits vergebene, mithin eingeführte Namen auf das Kino übertragen. Als Ott sein Kino im ehemaligen Kaufhaus Zur Glocke einbaute, übersetzte er diesen Namen ins Spanische und nannte sein Lichtspielhaus Campaneta; die Dreikaiser-Lichtspiele waren im ehemaligen Hotel Dreikaiserhof untergebracht. Andere Kinonamen, die sich bis in unsere Gegenwart hinein gehalten haben, wie Alhambra, Kosmos oder Universum verwiesen auf nichtalltägliche, die Phantasie anregende Zufluchtsorte, wohingegen Bezeichnungen wie Capitol, Colosseum und Olympia Bezüge zu Kultur und Götterwelt der Antike herstellten. Anklänge an einen bürgerlichen Bildungskanon sind hierbei nicht zu übersehen. Die Bezeichnung Faun-Palast zeigt, dass die Antike mit einem weiteren Glamourfaktor, nämlich Palast, verbunden werden konnte. Diese Kinonamen waren ebenso verbreitet wie

69 Zur Lichtreklame siehe Steidle: Kinoarchitektur, S. 286-292; allgemein zum elektrischen Licht im Stadtraum siehe den Beitrag von Lina Schröder in diesem Band.

70 Siehe Slidatke: Rummelplatz, S. 15. in der Frühzeit Bioskope, Elektrobiograph, Dedrophon und andere Bezeichnungen, hinter denen sich Markennamen für Projektoren oder sonstiges Kinoequipment verbargen. ${ }^{71}$

\section{Kinobesitzer}

Beim Kinonamen Rodera hingegen handelt es sich um eine auf den Namen des Erstbesitzers Alois Rodenstock anspielende Wortschöpfung. Diese Beobachtung führt zu den Kinobesitzern und ausdrücklich auch zu den Kinobesitzerinnen. Tatsächlich gehörte die Kinobranche zu jenen Unternehmenszweigen, in denen Frauen auch schon vor dem Ersten Weltkrieg in unterschiedlichen Konstellationen Fuß fassen konnten. Unter den rund 600 Personen, die während des Untersuchungszeitraums in der Dresdner Kinobranche greifbar sind, befanden sich 63 Frauen, die ihr Lichtspielhaus keinesfalls nur nach dem Witwen- und Töchterrecht betrieben. ${ }^{72}$ So gab es ,Unternehmerinnen' wie die geschäftstüchtige Franziska Böhme, die im Jahr 1911 das Welttheater kaufte und darüber hinaus im Folgejahr die Gründung des Germania in einem ihr gehörenden Wohn- und Geschäftshaus initiierte, wobei sie sich in diesem Fall mit der Pacht begnügte. Bei mindestens elf Kinos waren Frauen die Erstbesitzerinnen, wobei es sich in vier Fällen um Besitzerinnen von Gastwirtschaften

71 Siehe Liesegang: Namen und Baacke: Lichtspielhausarchitektur, Anm. 28.

72 Die Angaben sind lediglich sehr genaue Annäherungswerte, die auf der Sammlung Ott beruhen. Hier sind in einigen Fällen keine Vornamen genannt, außerdem kann bei Mehrfachnennungen in einigen Fällen nicht zweifelsfrei entschieden werden, ob es sich um eine oder um zwei Personen handelt. Zum Witwen- und Töchterrecht vergleiche Schötz: Unternehmerinnen, S. 55-64. 
beziehungsweise Ballsälen handelte, die ihr Unternehmen um einen Kinematographen erweiterten. Als Nachbesitzerinnen sind Frauen oftmals in der Konstellation ausgewiesen, dass der Ehemann das Gasthaus betrieb, in das die von der Ehefrau geführten Lichtspiele integriert waren.

Doch wer waren die Gründer der ersten ortsfesten Lichtspielhäuser? Die naheliegende Vermutung, es handele sich um die Besitzer der Wanderkinematographen, führt in die Irre. Nach dem Ende des Jahrmarktkinos blieben deren Besitzer im Regelfall weiterhin im Schaustellergewerbe tätig und wandten sich neuen Attraktionen zu. Auch Ott nennt nur wenige Schausteller, die versuchten, sich mit einem Kinematographen niederzulassen. Ein Sonderfall war dabei jener Kinobesitzer, der einerseits das Elektropanoptikum in der Prager Straße erwarb, andererseits aber ebenfalls in Dresden ein Kinozelt aufstellte, das Ott - eine Zeitungsmeldung zitierend - als Riesen-Prachtbau, der mit 1000 Glühlampen sowie weiteren 20 Bogenlampen beleuchtet war, beschrieb. ${ }^{73}$ Allerdings konstatierte er in diesen Fällen ein Scheitern nach nur wenigen Wochen, ohne allerdings spezifische Gründe zu benennen. Allerdings ist zu beachten, dass viele der frühen Kinos nur eine kurze Betriebsdauer besaßen. Ebenso könnte es sein, dass die Schausteller ähnlich wie zuvor in ihren Wanderkinos die Filme bis zum Verschleiß zeigten und nicht bedachten, dass ein ortsfestes Kino mit wöchentlich wechselnden Filmprogrammen um die Gunst des Publikums werben musste. Hier schließt als ein weiterer Sonderfall Heinrich Apel an, der

73 Sammlung Ott, Teil C, Blatt 9. als Inhaber eines Marionettentheaters von den Herausforderungen eines festen Schauspielbetriebes wusste und nicht nur selbst ein Kino betrieb, sondern nach Ott der zweite Vorsitzende im Kinobesitzerverein war. ${ }^{74}$

Eine eigene Berufsgruppe zwischen Wanderkinobesitzer und den Betreibern von ortsfesten Kinos bildeten bis in die 1930er-Jahre hinein Institutionen wie zum Beispiel das Arrangement Kosmographia, die Gesellschaft für wissenschaftliche und unterhaltende Kinematographie m.b.H. (Gefilge), der Deutsche Flottenverein oder der Konsum-Verein, die in unregelmäßigen Abständen Säle anmieteten, um hier eigens produzierte oder auch auf dem Markt vorhandene Filme mit wissenschaftlichen, technischen oder werberelevanten Inhalten zu zeigen. ${ }^{75} \mathrm{Zu}$ dieser Gruppe könnte man auch Schulfilmunternehmer zählen, zu denen die Sammlung Ott jedoch keinerlei Hinweise enthält. ${ }^{76}$

Doch in Ausnahmefällen konnte auch einem Schausteller der Umstieg ins Kinogeschäft längerfristig gelingen, wie Karl Paty bewies. Nachdem er zwischen 1900 und 1909 mit seinem Wanderkino auf der Dresdner Vogelwiese gastiert hatte, betrieb er unter anderem von 1911 bis 1921 ein festes Kino im Schillergarten, einem Ausflugslokal am Blauen Wunder, sowie ab 1917 einen Handel mit Kinozubehör. Vielleicht war es eine Folge der Hyperinflation, dass er zumindest sein Lichtspielhaus aufgab und ab 1924 zu seinen Wurzeln als Schausteller mit einem Wanderkinematographen und anderen

74 Siehe Jubiläums-Festschrift, S. 9.

75 Solche Lichtspiele nennt Sophie Döring in ihrem Beitrag in diesem Band temporäre Saalkinos.

76 Siehe den Aufsatz von Niklas Hertwig in diesem Band. 
Jahrmarktsattraktionen, etwa einer Schiffschaukel, zurückkehrte. ${ }^{77}$

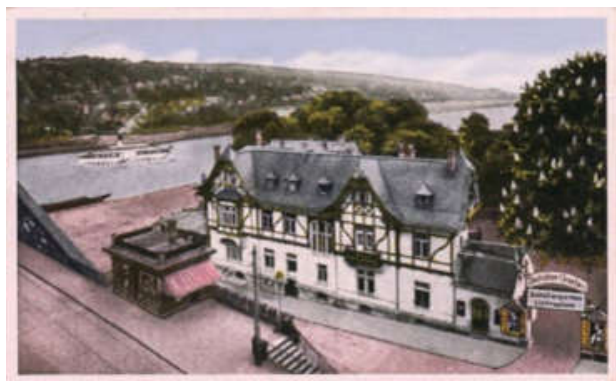

Abbildung 5: Eingang zum Kino im Restaurant Schillergarten, Postkarte 1939 (Quelle: https://altesdresden.de/ pics/blas/schi009c.jpg).

Dieser berufliche Werdegang verweist beispielhaft auf jene Eigenschaften, welche die frühen Kinobesitzer beziehungsweise -betreiber insgesamt besaßen. Als Quereinsteiger waren sie technikaffin, anpassungsfähig und bereit, ein beträchtliches wirtschaftliches Risiko einzugehen, zudem kannten sie sich idealiter in der Unterhaltungsbranche aus. Paradigmatisch ist erneut der Kinopionier Ott zu nennen: Die bereits genannte aufwendige Einrichtung des Campaneta nach dem Vorbild eines Theaters hatte seinen finanziellen Rahmen derart strapaziert, dass er sein Unternehmen ein knappes Jahr später veräußern musste - nicht weil er sich gegenüber einer überbordenden Konkurrenz nicht behaupten konnte, sondern weil er für den Schaden nach einem Filmbrand nicht aufkommen konnte. Feuerversicherungen für Kinos waren noch nicht erhältlich.
Doch aus welchem Personenkreis setzten sich die Besitzer beziehungsweise Betreiber der ortsfesten Kinos zusammen? Auch hier hilft die Sammlung Ott bei der Antwort. In den Blick gerät eine äußerst heterogene Personengruppe: Zu nennen sind etwa Hausbesitzer, die - wie der Kaufmann Richard Kremmler im Falle des Viktoria-Kinephon-Theater - einen Kinematographen in leerstehenden Ladenlokalen ihrer Wohnhäuser einrichteten. Eine andere Gruppe der Kinobetreiber bildeten jene Schank- und Gastwirte, die in mindestens 28 Wirtshäusern und in rund 30 Ballsälen Kinos installierten, in der Hoffnung, so den Umsatz anzukurbeln - was natürlich im Gegenzug bedeutete, dass viele Kinobesitzer Schanklizenzen für ihre Lichtspielhäuser beantragten sowie Verkaufs- und Imbissräume in ihren Kinos einrichteten, zumindest aber Verkaufsautomaten aufstellten. In der Konsequenz führte dies mitunter zu heftigen Auseinandersetzungen mit den Inhabern von Ladengeschäften, zu deren Verdruss der Verkauf von Schokolade und Gebäck im Kino an keine Ladenschließzeiten gebunden war. ${ }^{78}$

Eine interessante Gruppe unter den Kinobesitzern bildeten die Handwerker und Kaufleute. Einige von innen waren, so eine Forschungsmeinung, durch die zahlreichen neu entstandenen Warenhäuser in Bedrängnis geraten und mussten ihr Ladengeschäft aufgeben, weshalb sie ins Kinogeschäft einstiegen. Für andere hingegen war das Kino oftmals nur ein wirtschaftliches Standbein unter vielen. Ein Beispiel dafür ist Ernst Heinrich Georg Schulze, für den das Kinounternehmertum nur ein Berufsfeld neben

78 Siehe Stadtarchiv Dresden, Gewerbeamt, L0019. 
anderen war. ${ }^{79}$ In chronologischer Reihenfolge betrieb Schulze zwischen 1894 und 1923 eine Holz- und Kohlehandlung, er war Möbelspediteur, danach Besitzer eines Kinos im bürgerlichen Ortsteil Striesen, später eines Automobilhandels mit Reparaturwerkstatt sowie eines Kleinhandels mit Obst, Gemüse und Kartoffeln, schließlich arbeitete er als Makler und beendete sein Berufsleben mit der Herstellung und dem Vertrieb von Fahrrädern. Versuchte sich Schulze als Kleinunternehmer in mehreren Branchen, um seinen Lebensunterhalt zu verdienen, so verkörperte Edgar Gustav Wilhelm Schmidt einen anderen, finanzkräftigeren Typ des Kinobesitzers. ${ }^{80}$ Zwischen 1897 und 1930 war er Inhaber sowohl einer Luxus-Papier-Handlung als auch der Sächsischen Verlagsanstalt, 1919 übernahm er zusätzlich die chemische Fabrik Dr. Roscher. Seit dem Folgejahr engagierte er sich als Geldgeber im Kinogewerbe, die Akten des Gewerbeamtes verzeichnen ihn als persönlich haftenden Gesellschafter des Theaters am Fürstenplatz (Fü-Li), das er wenig später übernahm, um es an den Kaufmann Richard Schmitz weiterzuverkaufen. In den Jahren 1927 und 1929 folgten die Scala-Lichtspiele und das Gloria, die er beide verpachtete.

In der Frühzeit des Kinematographen gab es aber auch Kinounternehmer, die nicht nur ein oder mehrere Kinos, sondern auch weiterführende Betriebe im Kinogeschäft, etwa einen Film-Verleih, besaßen. Ein prominenter Vertreter war der Kaufmann Otto Carl Albrecht Dederscheck, der 1906 zunächst das bereits mehrfach erwähnte Dedrophon und bis Ende des

79 Siehe Stadtarchiv Dresden, Gewerbeamt, S7841.

80 Siehe Stadtarchiv Dresden, Gewerbeamt, S3988.
Folgejahres drei weitere Dedrophon-Kinos in Dresden und Freital gründete. Außerdem besaß er gemeinsam mit Fridolin Kretzschmar die Deutschen Kinematographen Werke in Dresden, wo er auch mit dem Glücksstern einen der ersten Filmverleihe gründete. Seine Geschäfte in Dresden endeten abrupt, als er im Jahr 1910 gemeinsam mit Kretzschmar zu fünf Monaten Gefängnis verurteilt wurde, nachdem beide pornografische Filme produziert und aufgeführt hatten. Danach ging Dederscheck in die Schweiz und gründete 1913 in Luzern die Film-Gesellschaft Express. Mitte der 1920er-Jahre wurde er schließlich in Berlin aktenkundig, wo er zum Missfallen der katholischen Kirche zwei Filme über Leben und Werk Papst Pius IX. vertrieb. ${ }^{81}$ Präsentiert die zugegeben etwas zwielichtige Figur Dederscheck einen Unternehmertypus, der in verschiedenen Sparten des Kinogeschäfts also Kinobesitz, Filmstudio, Kinematographenwerk und Verleih - tätig war, so gibt es ferner Beispiele für mittelständische Kinounternehmerdynastien, die über einen längeren Zeitraum verschiedene Kinos betrieben. Hier ist vor allem die Familie Schulze zu erwähnen, die über die doppelte Zäsur des Zweiten Weltkrieges und der Gründung der Deutschen Demokratischen Republik hinweg in verschiedenen Positionen im Dresdner Kinogeschäft tätig war. Willy Schulze kaufte 1919 mit dem Saxonia-Theater sein erstes Lichtspielhaus in Dresden, bis zum Ende

81 Zu Dederscheck siehe Otto Carl Albrecht Dederscheck, den Beitrag von Winfried Müller in diesem Band sowie Forster: Kino, S. 5. Zu den beiden Filmen vergleiche die verknüpften Dokumente in: http://194.242.233.158/denqPacelli/index. php?view=bio_layout\&bioConstraints[bylD]=yes\&bioConstraints[id] $=2325$. 
der Aufzeichnungen von Heinrich Ott folgten acht weitere Kinos, darunter die Musenhalle, der Dreikaiserhof sowie die Rädelsburg; seiner Frau Martha gehörten das Westend-Theater und die Walhalla. Gegen Kriegsende wurden seine Kinos zerstört, jedoch konnte er aus den Ruinen Teile der Kinotechnik und der Ausstattung retten, mit welchen er die Lichtspiele in DresdenWölfnitz eröffnete. Wohl 1948 wurde Schulze enteignet, noch im gleichen Jahr starb er. Sein Bruder arbeitete bis zu seinem Tod 1960 in den Volkslichtspielen Bünau als Vorführer. ${ }^{82}$ Neben diese Kinounternehmen traten bereits seit 1906 überregionale Betreiberfırmen und in Verbindung damit angestellte Kinodirektoren in Erscheinung. Hier ist etwa auf Max Flötner zu verweisen, der in den 1920er-Jahren für die Ufa zunächst in Leipzig, dann ab 1929 in Dresden Kinos wie das U.T. und das Universum leitete.

Der Abgleich der Besitzernamen aus der Sammlung Ott mit den Einträgen in den Adressbüchern verweist schließlich auf eine Besonderheit, die für andere Städte noch nicht registriert wurde: Viele Kinobetreiber waren exakt für jenen Zeitraum mit einem Dresdner Wohnort nachgewiesen, zu dem sie auch ihren Kinematographen betrieben, nicht aber für die Zeit davor und danach. In Verbindung damit, dass zahlreiche Lichtspielhäuser häufig ihre Besitzer wechselten, zeigt dies, wie bewegt der Kinomarkt im Untersuchungszeitraum war und welche Chancen er bot. Damit verbunden war auch eine hohe Mobilität der Besitzer. Offenkundig eröffnete der Besitz eines Lichtspielhauses die Chance, in der Großstadt Fuß zu fassen. Manche von

82 Zu Schulze siehe Fiedler: Kintopp, S. 161. diesen Kinobesitzern blieben mit einem neuen Beruf in Dresden sesshaft, auch nachdem sie mit ihrem Kino gescheitert waren, andere sind dagegen nicht mehr nachweisbar. Ob die fraglichen Kinobesitzer jedoch in der Zeit vor beziehungsweise nach ihrer Dresdner Tätigkeit als Kinobetreiber in einer der umliegenden Städte ein Lichtspielhaus betrieben, kann derzeit nicht beantwortet werden.

\section{Zusammenfassung}

Bei der Etablierung des Kinos als Leitmedium der neuen Massenkultur im ersten Drittel des 20. Jahrhunderts ordnet sich Dresden in eine allgemeine Entwicklung ein, die in allen Großstädten im Wesentlichen synchron ablief. Das Verdienst der Sammlung Ott besteht darin, dass sie Daten zu 153 Kinos sowie die Namen von rund 460 Personen der Dresdner Kinogeschichte dokumentiert. Zunächst erlauben es die Aufzeichnungen Otts, in chronologischer Reihenfolge eine Kinotopografie zu erstellen, in der nicht nur die genauen Standorte der einzelnen Lichtspielhäuser im Stadtplan verortet sind, sondern auch räumliche und soziokulturelle Beziehungen zu weiteren Orten des Massenvergnügens wie Theatern, Ballhäusern oder Kneipen verdeutlicht werden. Die lückenlose Auflistung der Besitzer und Betreiber erlaubt außerdem weitere Rückschlüsse sowohl auf Kinobesitzerdynastien als auch in Kombination mit den Archivalien des Gewerbeamtes auf die Erwerbsbiografie einzelner Kinounternehmer. Damit werden zugleich Verbindungen zu anderen Unternehmenszweigen der urbanen Freizeitkultur deutlich. Die Eröffnungsannoncen der Kinos und in 
die Sammlung Ott eingestreute Kurztexte liefern schließlich weiterführende Informationen zur lokalen Kinokultur sowie zur Technik- und Verwaltungsgeschichte.

Hinweise auf mögliche Dresdner Spezifika liefert Heinrich Ott freilich bestenfalls en passant: Sie liegen darin, dass die Elbestadt nicht nur großstadttypisch eine Kinometropole war, sondern mit den Ernemann-Werken auch ein Zentrum der Kinotechnik!

\section{Linksammlung}

Alle Zugriffe am 24.06.2020.

http://194.242.233.158/denqPacelli/index.php?view=bio_ layout\&bioConstraints[bylD]=yes\&bioConstraints[id] $=2325$ https://www.dresden.de/media/pdf/statistik/Dresdner_ Zahlen_aktuell_16_2009_08.pdf

https://www.filmportal.de/film/der-rosenkavalier_caa73c 6fb943488196547819420d0739

https://www.neustadt-ticker.de/57108/aktuell/ schauburg-grundungsurkunde-gefunden

\section{Archivalien}

\section{Dresden, Stadtarchiv}

Bestand 2.3.9: Gewerbeamt

L0019, Film L0002: Paul Reinhold Lachmann.

P0473, Film P0017: Karl Paty.

S3988, Film S0148 und S 0149: Edgar Gustav Wilhelm Schmidt.

S7841, Film S0290: Ernst Heinrich Georg Schulze.

Bestand 17.2: Zeitgeschichtliche Sammlungen, 17.2.10: Sammlung Heinrich Ott zur Geschichte der Dresdner Kinematographie.

Teil C: Die Kinematographie als Schaustellung
Teil F: Dresdner Filmverleiher

Teil J: Gründung des Vereins der Dresdner Kinobesitzer

Teil M: Chronologisches Verzeichnis aller Filme von 1906 bis 1936

\section{Literatur und Quellen}

Adreßbuch für Dresden und seine Vororte, 1906-

1943/44. URN: urn:nbn:de:bsz:14-db-id32253136Z6

Bekanntmachung, die Veranstaltung kinematographischer Vorführung betreffend. Dresden, 8. Mai 1909.

Chronologisches Verzeichnis der von Victor Klemperer 1919-1932 gesehenen und in den Tagebüchern genannten bzw. besprochenen Filme, in: Klemperer Online. Tagebücher 1918-1959. Hrsg. v. Walter Nojowski/Christian Löser., Berlin 2019; URL: https://db-degruyter-com. wwwdb.dbod.de/staticfiles/content/dbsup/KLEMP_8_ KinoFilme.pdf.

Der erste Kinotheater-Bau, in: Kinema 3(1913)11; URL: http://doi.org/10.5169/seals-719190.

Jubiläums-Festschrift des Mitteldeutschen Bezirksverbands: Verein der Lichtspieltheaterbesitzer von Dresden u[nd] Umg[ebung] e[ingetragener] V[erein] (Im Reichsverband deutscher Lichtspieltheaterbesitzer e[ingetragener] V[erein]) anläßlich seines 20jährigen Bestehens 1909, 14. Mai 1929.

Otto Carl Albrecht Dederscheck, in: ,Kritische Online-Edition der Nuntiaturberichte Eugenio Pacellis (1917-1929)', Kurzbiografie Nr. 2325; URL: www.pacelli-edition.de/ gnd/116044276.

Rolf-Peter Baacke: Lichtspielhausarchitektur in Deutschland. Von der Schaubude bis zum Kinopalast, Berlin 1982.

Astrid Brosch: Kinobauten der 1950er Jahre im geteilten Deutschland, München 2003.

Paul Dienstag/Alexander Elster: Handbuch des deutschen Theater-, Film-, Musik- und Artistenrechts, Berlin 1932. 
Heinz Fiedler: Vom Kintopp zum modernen Lichtspielhaus, in: Stadtmuseum Dresden (Hg.): Dresdner Geschichtsbuch, Band 1, Dresden 1995, S. 151-169.

Wolfgang Flügel: 1918 als Achsenjahr der Massenkultur. Kino, Filmindustrie und Filmkunstdiskurse in Dresden. Ein Werkstattbericht, in: Denkströme. Journal der Sächsischen Akademie der Wissenschaften Leipzig, Heft 21 (2019), S. 137-154.

Ralf Forster: Vom „Kino" und seinen Nachfolgern. Dresden als Zentrum der Filmtechnik, in: Dresdner Hefte. Beiträge zur Kulturgeschichte 23 (2005), Heft 82, S. 3-12.

Max Frank: Was man beim Kauf eines Lichtspieltheaters zu beachten hat!, in: Kinema 5 (1915) Heft 2; URL: http:// doi.org/10.5169/seals-719193.

Joseph Garncarz: Medienwandel, Konstanz/München 2016.

Joseph Garncarz: Öffentliche Räume für Filme. Zur Etablierung des Kinos in Deutschland, in: Corinna Müller/ Harro Segeberg (Hg.): Kinoöffentlichkeit (1895-1920). Entstehung-Etablierung-Differenzierung, Marburg 2008 , S. 31-43.

Joseph Garncarz: Über die Entstehung der Kinos in Deutschland 1896-1914, in: KINtop. Jahrbuch zur Erforschung des frühen Films 11 (2002), S. 144-158.

Andrea Haller: Frühes Kino zwischen Stadt und Land. Einige Überlegungen zum Verhältnis von Kinoprogrammgestaltung, Kinopublikum und moderner Stadterfahrung vor 1914, in: Tobias Becker/Anna Litmann/Johanna Niedbalski (Hg.): Die tausend Freuden der Metropole. Vergnügungskultur um 1900, Bielefeld 2011, S. 229-256.

Gabriele Klunkert: Schaustellungen und Volksbelustigungen auf Leipziger Messen des 19. Jahrhunderts. Eine wirtschafts- und sozialgeschichtliche Untersuchung, Göttingen 2010

Ralf Kukula/Volker Helas: Ballhäuser in Dresden, 2. überarbeitete Auflage Dresden 1997.

Jasmin Lange: Der deutsche Buchhandel und der Siegeszug der Kinematographie 1895-1933. Reaktionen und strategische Konsequenzen, Wiesbaden 2010.
Paul Liesegang: Wer zählt die Namen? Auch ein Beitrag zur Entwicklungsgeschichte des Kinematographen, in: Jahrbuch für Photographie und Reproduktionstechnik 28 (1914), S. 19-22; URL: https://doi.org/10.11588/ diglit.45030.8.

Kaspar Maase: Bewegliche Grenzen. Überlegungen zur Bestimmung von Populärkultur in der Weimarer Republik, in: Jessica Nitzsche/Nadine Werner (Hg.): Populärkultur, Massenmedien, Avantgarde 1919-1933, München 2012, S. $21-36$

Kaspar Maase: Grenzenloses Vergnügen. Der Aufstieg der Massenkultur 1850-1970, 3. Auflage Frankfurt am Main 2001.

Corinna Müller: Frühe deutsche Kinomatographie. Formale, wirtschaftliche und kulturelle Entwicklungen, 1907-1912, Stuttgart 1994.

Corinna Müller/Harro Segeberg: „Öffentlichkeit" und „Kinoöffentlichkeit". Zum Hamburger Forschungsprogramm, in: Corinna Müller/Harro Segeberg (Hg.): Kinoöffentlichkeit (1895-1920): Entstehung, Etablierung, Differenzierung, Marburg 2008, S. 7-30.

Winfried Müller/Sophie Döring: „Der Kino“, „die Films“. Ein neues Medium kommt in Dresden an, in: Dresdner Hefte. Beiträge zur Kulturgeschichte 37 (2019), Heft 137, S. 71-79.

Carola Neumann: Von der Schaubude zum Kristallpalast. Kinoarchitektur in Dresden, in: Dresdner Hefte. Beiträge zur Kulturgeschichte 23 (2005), Heft 82, S. 25-33.

Ray Oldenburg: The Great Good Place. Cafés, Coffee Shops, Community Centers, Beauty Parlors, General Stores, Bars, Hangouts and how they Get you through the Day, New York 1989.

Rudolf Pabst: Das deutsche Lichtspieltheater in Vergangenheit, Gegenwart und Zukunft, Berlin 1926.

Esther Sabelus/Jens Wietschorke: Einleitung, in: Esther Sabelus/Jens Wietschorke: Die Welt im Licht. Kino im Berliner Osten, Berlin 2015, S. 7-10.

Susanne Schötz: Unternehmerinnen im 19. Jahrhundert. Das Beispiel von Leipziger Handelsfrauen, in: Ulrich Heß/Michael Schäfer ( $\mathrm{Hg}$.): Unternehmer in Sachsen. 
Aufstieg - Krise - Niedergang - Neubeginn, Leipzig 1998,

S. 55-64.

Anne Slidatke: Vom Rummelplatz in die Innenstadt, in: kunsttexte.de 10(2015), S. 1-19.

L.W. Stäubli: Kino und Grundstücksmarkt, in: Kinema 3 (1913) Heft 13; URL: http://doi.org/10.5169/seals-719242.

Sabine Steidle: Kinoarchitektur als Chiffre für großstädtisches Leben und Modernität in der Weimarer Republik, in: Gabriele B. Clemens u.a. (Hg.): Städtischer Raum im Wandel. Modernität-Mobilität-Repräsentationen, Berlin 2011, S. 281-300.

Bettina Vaupel: Eine kleine Kulturgeschichte des Kinos. „Zweimal Sperrsitz, bitte!“, in: Monumente. Magazin für Denkmalkultur in Deutschland; URL: https://www.monumente-online.de/de/ausgaben/2008/2/zweimal-sperrsitz-bitte.php.

Kirsten Vincenz/Wolfgang Hesse ( $\mathrm{Hg}$.): Fotoindustrie und Bilderwelten. Die Heinrich-Ernemann-AG für Camerafabrikation in Dresden 1889-1926, Bielefeld/Leipzig 2008.

Jens Wietschorke: Die Soziale Arbeitsgemeinschaft Berlin-Ost und der bürgerliche Blick auf das moderne Massenvergnügen, in: Esther Sabelus/Jens Wietschorke: Die Welt im Licht. Kino im Berliner Osten 1900-1930, Berlin 2015, S. 11-40

Yong Chan Choy: Inszenierung der völkischen Filmkultur im Nationalsozialismus. Der internationale Filmkongress Berlin 1935, Dissertation TU Berlin 2006.

Carola Zeh: Lichtspieltheater in Sachsen. Entwicklung, Dokumentation und Bestandsanalyse, Hamburg 2007. 
A.G./AG

$\mathrm{AHL}$

Anm.

Art.

BArch

BayHStA

Bd.

Best.

$\mathrm{Bl}$.

BLHA

BPA

bzW.

CDU

CFC

Co.

DCP

DDR

DEFA

Dir.

DJ

DNN

Dr.

DVD

EFKA

e.V.

FDGB

FDP

FF

$\mathrm{GmbH}$

GP

$\mathrm{H}$.

$\mathrm{Hg}$.

$\mathrm{HO}$

HStA Dresden

ISGV

$\mathrm{Jg}$.

KPD

$\mathrm{LMZH}$

LRA

LVR-ILR

MA GAP

MK

$\mathrm{mm}$

MNN

$\mathrm{Nr}$.

NS

NSDAP

ntv
Paragraf

Pfennig(e)

und

dreidimensional

Abbildung

Allgemeine Elektricitäts-Gesellschaft

Aktiengesellschaft

Archiv Hansestadt Lübeck

Anmerkung

Artikel

Bundesarchiv

Bayerisches Hauptstaatsarchiv München

Band

Bestand

Blatt

Brandenburgisches Landeshauptarchiv

Presse- und Informationsamt der Bundesregierung

beziehungsweise

Christlich-Demokratische Union

Corso Film Casino

Compagnie

Digital Cinema Packages

Deutsche Demokratische Republik

Deutsche Film AG

Direktor

Discjockey

Dresdner Neueste Nachrichten

Doktor

Digital Video Disc/Digital Versatile Disc

Frankfurter Kammerlichtspiele

eingetragener Verein

Freier Deutscher Gewerkschaftsbund

Freie Demokratische Partei

Filmförderungsanstalt

Gemeinschaft mit beschränkter Haftung

Gewerbepolizei

Heft

Herausgeber

Handelsorganisation

Sächsisches Staatsarchiv - Hauptstaatsarchiv Dresden

Institut für Sächsische Geschichte und Volkskunde Dresden

Jahrgang

Kommunistische Partei Deutschlands

Landesmedienzentrum Hamburg

Landratsamt

Landschaftsverband Rheinland-Institut für Landeskunde und Regionalgeschichte Marktarchiv Garmisch-Partenkirchen

Kultusministerium

Millimeter

Münchner Neueste Nachrichten

Nummer

Nationalsozialistisch, auch: Nationalsozialismus

Nationalsozialistische Arbeiterpartei Deutschlands

Fernsehnachrichtensender 


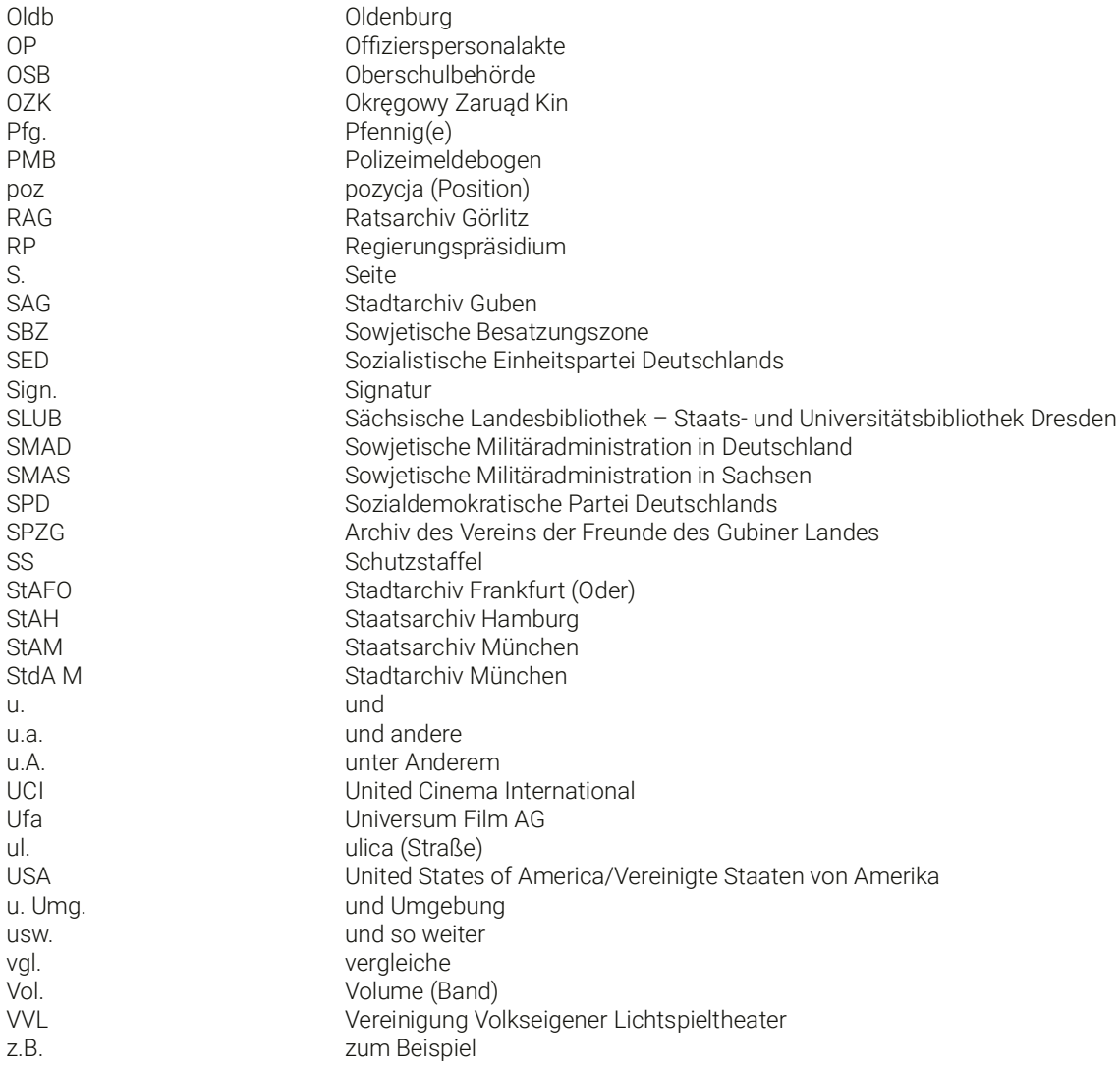

